\title{
The circumburst density profile around GRB progenitors: a statistical study ${ }^{\star}$
}

\author{
S. Schulze ${ }^{1}$, S. Klose ${ }^{2}$, G. Björnsson ${ }^{1}$, P. Jakobsson ${ }^{1}$, D. A. Kann ${ }^{2}$, A. Rossi ${ }^{2}$, T. Krühler ${ }^{3,4}$, \\ J. Greiner ${ }^{3}$, and P. Ferrero ${ }^{5,6}$
}

${ }^{1}$ Centre for Astrophysics and Cosmology, Science Institute, University of Iceland, Dunhagi 5, 107 Reykj
e-mail: steve@raunvis.hi .is
2 Thüringer Landessternwarte Tautenburg, Sternwarte 5, 07778 Tautenburg, Germany
3 Max-Planck-Institut für Extraterrestrische Physik, Giessenbachstraße 3, 85748 Garching, Germany
4 Universe Cluster, Technische Universität München, Boltzmannstraße 2, 85748 Garching, Germany
5 Instituto de Astrofísica de Canarias (IAC), 38200 La Laguna, Tenerife, Spain
6 Departamento de Astrofísica, Universidad de La Laguna (ULL), 38205 La Laguna, Tenerife, Spain

Received 13 August 2010 / Accepted 21 October 2010

\section{ABSTRACT}

\begin{abstract}
According to our present understanding, long gamma-ray bursts (GRBs) originate from the collapse of massive stars, while short bursts are caused by to the coalescence of compact stellar objects. Because the afterglow evolution is determined by the circumburst density profile, $n(r)$, traversed by the fireball, it can be used to distinguish between a constant density medium, $n(r)=$ const., and a free stellar wind, $n(r) \propto r^{-2}$. Our goal is to derive the most probable circumburst density profile for a large number of Swiftdetected bursts using well-sampled afterglow light curves in the optical and X-ray bands. We combined all publicly available optical and Swift/X-ray afterglow data from June 2005 to September 2009 to find the best-sampled late-time afterglow light curves. After applying several selection criteria, our final sample consists of 27 bursts, including one short burst. The afterglow evolution was then studied within the framework of the fireball model. We find that the majority (18) of the 27 afterglow light curves are compatible with a constant density medium (ISM case). Only 6 of the 27 afterglows show evidence of a wind profile at late times. In particular, we set upper limits on the wind termination-shock radius, $R_{\mathrm{T}}$, for GRB fireballs that are propagating into an ISM profile and lower limits on $R_{\mathrm{T}}$ for those that were found to propagate through a wind medium. Observational evidence for ISM profiles dominates in GRB afterglow studies, implying that most GRB progenitors might have relatively small wind termination-shock radii. A smaller group of progenitors, however, seems to be characterised by significantly more extended wind regions.
\end{abstract}

Key words. gamma-ray burst: general - ISM: structure - radiation mechanisms: non-thermal

\section{Introduction}

Starting with the discovery of the gamma-ray burst-supernova (GRB-SN) association GRB 980425/SN 1998bw (Galama et al. 1998; Iwamoto et al. 1998; Sollerman et al. 2000), there is by now convincing evidence that the progenitors of long GRBs are massive stars exploding as type Ic SNe (for a review see Woosley $\&$ Bloom 2006). Within this picture, the optical light observed after a long GRB is the superposition of the afterglow light, a supernova component, and light from the underlying host galaxy (plus potential additional radiation components at very early times, which we will not consider here). Phenomenologically, this immediately unveils two observing strategies to reveal a massive-star origin of a GRB: $(i)$ via the detection of a late-time SN bump (method $i$ ) in the optical light curve (e.g., Reichart 1999; Galama et al. 2000; Dado et al. 2002; Zeh et al. 2004) and (ii) by the spectroscopic confirmation (method ii) of associated supernova light (the best case so far being GRB 030329: Hjorth et al. 2003; Kawabata et al. 2003; Matheson et al. 2003; Stanek et al. 2003). In addition to both observing strategies, there are two further methods by which a massive-star origin can be revealed. Some afterglow spectra showed blue-shifted absorption

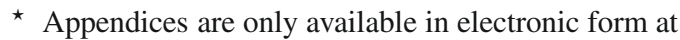
http: //www . aanda.org line systems (method iii), which can be understood as signatures from the expanding pre-explosion wind escaping from the GRB progenitor (e.g., Mirabal et al. 2003; Schaefer et al. 2003; Klose et al. 2004; Starling et al. 2005; Berger et al. 2006; Fox et al. 2008; Castro-Tirado et al. 2010). Some authors, however, notice that several properties of the putative blue-shifted absorption line systems disagree with the expectations from Wolf-Rayet (WR) winds, e.g., line widths, ionisation levels and metallicities (Chen et al. 2007; Prochaska et al. 2007; Fox et al. 2008). Finally (method $i v$ ), the circumburst medium determines the spectral and temporal evolution of the afterglow, allowing us to discern between a constant-density medium, $n(r)=$ const., and a wind medium, $n(r) \propto r^{-2}$ (Sari et al. 1998; Chevalier \& Li 1999, 2000). This method was successfully applied in, e.g., Starling et al. (2009) and Curran et al. (2010).

Naturally, these various approaches have their observational advantages and disadvantages. While method (ii) can provide the strongest observational evidence for a massive-star origin of the GRB under consideration, it can only be applied to the nearest and hence brightest events up to a redshift of about 0.5. Even 13 years after the first discovery of an afterglow (Costa et al. 1997; van Paradijs et al. 1997), i.e., after more than 500 GRBs with detected (X-ray, optical, radio) afterglow light ${ }^{1}$, secure evidence

${ }^{1}$ http://www . mpe.mpg.de/ jcg/grbgen.html 
for a spectroscopically associated $\mathrm{SN}$ was only reported for roughly $1 \%$ of all events (GRBs 980425: Galama et al. 1998; 030329: Hjorth et al. 2003; Kawabata et al. 2003; Matheson et al. 2003; Stanek et al. 2003; 031203: Malesani et al. 2004; 060218: Ferrero et al. 2006; Mirabal et al. 2006; Modjaz et al. 2006; Pian et al. 2006; Sollerman et al. 2006; 081007: Della Valle et al. 2008; 100316D: Chornock et al. 2010; Starling et al. 2010). Contrary to this approach, method $(i)$ can basically reveal a SN component up to a redshift of about 1 (Zeh et al. 2004), assuming a non-extinguished SN 1998bw as a template (the most distant SN bump was found for GRB 000911 at $z=1.06$; Masetti et al. 2005). At notably higher redshifts a GRB-SN becomes too faint to be discovered even with $8 \mathrm{~m}$-class optical telescopes because of line blanketing (e.g., Filippenko 1997).

In principle, methods (iii) and (iv) do not have redshift constraints, because both rely on the observation of the afterglow and not on the (expected) SN component. In addition, method (iv) splits into different approaches and basically works for the optical and X-ray band in the same way. Moreover, it can even be applied to the most distant GRBs, which are already affected by Lyman dropout in the optical bands. Its main disadvantage is that it usually requires substantial observational efforts. In particular, it relies on the measurement of the light-curve evolution, including the determination of the spectral energy distribution (SED).

In this paper, applying method (iv), we use late-time data of afterglows detected by Swift to tackle the question of the preferred density profiles in a statistical sense. Our goal is to combine all publicly available optical data with Swift/XRT data in order to determine the corresponding circumburst density profile, $n(r) \propto r^{-k}$. Qualitatively, this splits into either a constantdensity medium (hereafter referred to as the interstellar medium or ISM) $(k=0)$ or a free wind profile $(k=2)$. While the complex wind history of an evolved massive star might produce density profiles different from the ideal case $(k=0,2$; Crowther 2007), in general the data do not allow for a more accurate determination of $k$, but only to distinguish between these two cases.

It should be stressed that the intention of our study is not to provide ultimate conclusions on the density profiles found for individual bursts. Instead, it is meant as a statistical approach using bursts with the best available X-ray as well as optical data. The questions we want to address are: (1) What is, in a statistical sense, the preferred circumburst density profile? (2) What is the ratio between events with ISM and with wind profiles? (3) What does this tell us about the typical radius of the wind termination shock that is expected to exist in the ambient medium surrounding a long burst GRB progenitor?

Throughout the paper we use the convention $F_{v}(t) \propto t^{-\alpha} v^{-\beta}$ for the flux density, where $\alpha$ is the temporal slope and $\beta$ is the spectral slope. All errors are $1 \sigma$ uncertainties unless noted otherwise.

\section{Data selection}

\subsection{Data gathering}

The optical data were taken from a photometric database maintained and updated by one of the co-authors (D.A.K.). In addition, we added data for GRB 090726 from Šimon et al. (2010), Fatkhullin et al. (2009), Haislip et al. (2009), Kelemen (2009), Landsman \& Page (2009), Sakamoto et al. (2009) and Volnova et al. (2009). The properties of the optical afterglow sample, the data gathering and the deduced SEDs of the afterglows are discussed in Kann et al. (2006, 2008, 2010). Furthermore, we compared their SED results with the work of Schady et al. (2007, 2010). The latter authors added $X$-ray data to model the spectral energy distributions from roughly $1 \mathrm{eV}$ to $10 \mathrm{keV}$. Finally, to create a denser light-curve coverage, all non- $R_{\mathrm{C}}$ band data in each light curve were shifted to the $R_{\mathrm{C}}$ band. In doing this, we used the colours of the SED, assuming no spectral evolution and omitting data where clear colour evolution was evident.

The X-ray data were retrieved from the Swift data archive and the light curves from the Swift light-curve repository (version February 2010) updated and maintained by Evans et al. (2007, 2009). Following Nousek et al. (2006), we reduced the data with the software package HeaSoft $6.6 .1^{2}$ together with the calibration file version $v \mathbb{Q} 11^{3}$. Furthermore, we applied the methods detailed in Moretti et al. (2005), Romano et al. (2006), and Vaughan et al. (2006) to reduce pile-up affected data. We extracted SEDs at different epochs with approximately 500 background subtracted counts, to check for spectral evolution. If the properties of the SED (spectral slope and absorption) did not evolve, we extracted a new SED from the maximum possible time interval. In addition, we used the Tübingen absorption model by Wilms et al. (2000) and their interstellar-medium metal abundance template. The Galactic absorption was fixed to the weighted mean based on Kalberla et al. (2005). We included the Chandra light curve of GRB 051221A from Burrows et al. (2006).

\subsection{Sample definition and light-curve fitting}

Among all Swift GRBs observed until September 2009, we selected from our photometric database, which contains long and short bursts, those 90 bursts that have an optical and an X-ray afterglow as well as a measured spectroscopic or a photometric redshift. From these we selected those bursts with the bestsampled optical and X-ray afterglow light curves as follows.

First, we required information on the spectral slope of the afterglow in the X-ray band. We derived this information from publicly available data. In the optical bands, however, multiband data are usually not available. In these cases we used the results from Kann et al. (2008, 2010) and Schady et al. (2007, 2010), with the latter being based on optical-to-X-ray SED fits.

Second, we required that an afterglow light curve can be fitted with a multiply broken power-law and is not dominated by flares or bad data sampling. After excluding time intervals affected by flares, we fitted the light curves with a smoothly broken power-law of the order $m$ (see Appendix A for its definition) with a Simplex and a Levenberg-Marquardt algorithm (Press et al. 2007). Furthermore, we transformed $R_{\mathrm{c}}$-band light curves to flux densities with the zero-point definition of Bessell (1979). For the X-ray regime we followed Gehrels et al. (2008); the flux density, $F_{v, \mathrm{x}}$, in $\mu \mathrm{Jy}$ at the frequency $v_{\mathrm{x}}$ is then given by

$$
F_{v, \mathrm{x}}=4.13 \times 10^{11} \frac{\left(1-\beta_{\mathrm{x}}\right) F_{\mathrm{x}}}{(10 \mathrm{keV})^{1-\beta_{\mathrm{x}}}-(0.3 \mathrm{keV})^{1-\beta_{\mathrm{x}}}} E_{\mathrm{x}}^{-\beta_{\mathrm{x}}},
$$

where $\beta_{\mathrm{x}}$ is the spectral slope in the $\mathrm{X}$-ray band, $F_{\mathrm{X}}$ is the measured flux in the $0.3-10 \mathrm{keV}$ range in units of $\mathrm{erg} \mathrm{cm}^{-2} \mathrm{~s}^{-1}$ and the reference energy $E_{\mathrm{x}}$ is given in $\mathrm{keV}$. For all bursts we chose the logarithmic mean between $0.3 \mathrm{keV}$ and $10 \mathrm{keV}$ as a reference, i.e., $E_{\mathrm{x}}=1.73 \mathrm{keV}\left(v_{\mathrm{x}}=4.19 \times 10^{17} \mathrm{~Hz}\right)$. The numerical constant converts the energy in units of $\mathrm{keV}$ to a frequency in units of $\mathrm{Hz}$ and the flux density from $\mathrm{erg} \mathrm{cm}^{-2} \mathrm{~s}^{-1} \mathrm{~Hz}^{-1}$ to $\mu \mathrm{Jy}$.

\footnotetext{
2 http://heasarc.gsfc.nasa.gov/docs/software/lheasoft

3 heasarc.gsfc.nasa.gov/docs/heasarc/caldb/swift
} 
Table 1. Summary of the considered afterglow models.

\begin{tabular}{|c|c|c|c|}
\hline Spectral regime & $\beta_{\mathrm{x}}-\beta_{\mathrm{opt}}$ & $\alpha_{\mathrm{x}}-\alpha_{\mathrm{opt}}$ & $F_{\gamma, \mathrm{opt}} / F_{\gamma, \mathrm{x}}$ \\
\hline \multicolumn{4}{|c|}{ Spherical expansion } \\
\hline$v_{\mathrm{c}}<v_{\mathrm{opt}}<v_{\mathrm{x}}$ & 0 & 0 & $\left(v_{\mathrm{opt}} / v_{\mathrm{x}}\right)^{-p / 2}$ \\
\hline$v_{\mathrm{opt}}<v_{\mathrm{c}}<v_{\mathrm{x}}$ & $1 / 2$ & $\pm 1 / 4$ & $v_{\mathrm{opt}}^{-(p-1) / 2} v_{\mathrm{x}}^{p / 2} v_{\mathrm{c}}^{-1 / 2}(t)$ \\
\hline$v_{\mathrm{opt}}<v_{\mathrm{X}}<v_{\mathrm{c}}$ & 0 & 0 & $\left(v_{\mathrm{opt}} / v_{\mathrm{X}}\right)^{-(p-1) / 2}$ \\
\hline \multicolumn{4}{|c|}{ Jet with sideways expansion } \\
\hline$v_{\mathrm{c}}<v_{\mathrm{opt}}<v_{\mathrm{x}}$ & 0 & 0 & $\left(v_{\mathrm{opt}} / v_{\mathrm{x}}\right)^{-p / 2}$ \\
\hline$v_{\mathrm{opt}}<v_{\mathrm{c}}<v_{\mathrm{x}}$ & $1 / 2$ & 0 & $v_{\mathrm{opt}}^{-(p-1) / 2} v_{\mathrm{x}}^{p / 2} v_{\mathrm{c}}^{-1 / 2}$ \\
\hline$v_{\mathrm{opt}}<v_{\mathrm{x}}<v_{\mathrm{c}}$ & 0 & 0 & $\left(v_{\mathrm{opt}} / v_{\mathrm{x}}\right)^{-(p-1)}$ \\
\hline \multicolumn{4}{|c|}{ Jet without sideways expansion } \\
\hline$v_{\mathrm{c}}<v_{\mathrm{opt}}<v_{\mathrm{x}}$ & 0 & 0 & $\left(v_{\mathrm{opt}} / v_{\mathrm{x}}\right)^{-p / 2}$ \\
\hline$v_{\mathrm{opt}}<v_{\mathrm{c}}<v_{\mathrm{x}}$ & $1 / 2$ & $\pm 1 / 4$ & $v_{\mathrm{opt}}^{-(p-1) / 2} v_{\mathrm{x}}^{p / 2} v_{\mathrm{c}}^{-1 / 2}(t)$ \\
\hline$v_{\mathrm{opt}}<v_{\mathrm{x}}<v_{\mathrm{c}}$ & 0 & 0 & $\left(v_{\text {opt }} / v_{\mathrm{x}}\right)^{-(}$ \\
\hline
\end{tabular}

Notes. Theoretical differences in the spectral and temporal slopes as well as the flux-density ratio depend on the position of the cooling frequency, $v_{\mathrm{c}}$, with respect to the optical and the X-ray band $\left(v_{\mathrm{opt}}, v_{\mathrm{x}}\right.$, respectively; valid for an electron index, $p$, of larger than 2; e.g., Zhang \& Mészáros 2004; Panaitescu 2007). Depending on the circumburst medium the positive (ISM) or negative (wind) solution for $\alpha_{\mathrm{x}}-\alpha_{\mathrm{opt}}$ applies.

Third, once power-law segments had been defined in the afterglow light curves, we excluded from further studies those bursts where the difference in the late-time decay slopes between the optical and the X-ray band could not be explained within the framework of the fireball model (Table 1); i.e., the difference in decay slopes, $\alpha_{\mathrm{x}}-\alpha_{\mathrm{opt}}$, was larger than $1 / 4$ within $3 \sigma$. Because of this, a strict criterion for the beginning of the late-time evolution of an afterglow cannot be given. Evidence for an observed canonical light curve does not exist in the optical (Kann et al. 2008, 2010; Panaitescu \& Vestrand 2010), but may exist in the X-rays (e.g., Nousek et al. 2006; Zhang et al. 2006; Evans et al. 2009). Therefore, as an operational definition an afterglow is in its late-time phase when its temporal and spectral evolution can be explained by the fireball model from a particular time after the corresponding GRB.

After applying these selection criteria, about half of the 90 bursts had to be rejected owing to bad sampling, poor data quality, flares, and other peculiarities. Another quarter had to be rejected because of $\left|\alpha_{\mathrm{x}}-\alpha_{\text {opt }}\right|>1 / 4$ within $3 \sigma$. All data were then corrected for host extinction in the optical, $A_{V}^{\text {host }}$, if needed, and for Galactic and host absorption in the X-ray band, $N_{\mathrm{H}}^{\text {host }}$.

In total, 27 afterglows passed these selection criteria. The sample consists of 25 long and one short GRB (GRB 051221A), and one controversial event (GRB 060614) in terms of the short/long classification scheme. Their input data are summarised in Tables B.1 and B.2.

\section{Results and discussion}

\subsection{The circumburst medium density profile}

\subsubsection{Identifying the circumburst medium}

As a first step in the identification of the circumburst medium, we defined nine possible spectral and dynamical regimes (Table 2). The spectral regimes separate according to the position of the cooling frequency with respect to the observer frame,
Table 2. The closure relations combining the temporal decay slope $\alpha$ and the spectral slope $\beta$ (adopted from Zhang \& Mészáros 2004; and Panaitescu 2007; valid for $p>2$ ).

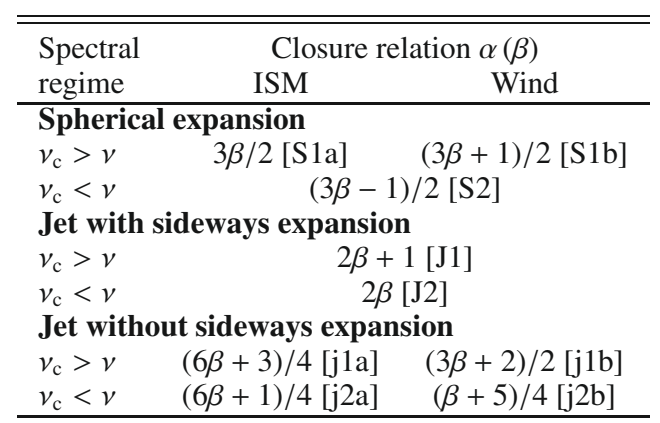

Notes. Abbreviation used here for a certain model is given in brackets (adopted from Panaitescu 2007; (S, J, j)=(spherical expansion, jet with sideways expansion, jet without lateral spreading $),(1,2)=\left(v<v_{\mathrm{c}}\right.$, $\left.v>v_{\mathrm{c}}\right),(\mathrm{a}, \mathrm{b})=(\mathrm{ISM}$, wind) $)$. Entries extending over two columns are valid for both media.

while the dynamical regimes distinguish between a spherical and a jetted evolution.

To derive the most probable density profile into which a GRB jet propagates, we proceeded in the following way. The main criterion was that the result agrees with the optical as well as with the X-ray data. In doing so, we first analysed the closure relations for the nine models (Table 2) in the optical and X-ray bands and selected only those relations (models) which were fulfilled within $3 \sigma$. Second, we computed the difference in the decay slopes, $\alpha_{\mathrm{x}}-\alpha_{\mathrm{opt}}$, and distinguished between the models according to the three possible cases $-1 / 4,0,+1 / 4$ (Table 1 ), again within $3 \sigma$. Third, if possible, we took into account the difference in the spectral slope, $\beta_{\mathrm{x}}-\beta_{\text {opt }}$, which is either 0 or $1 / 2$ (see Table 1). If this criterion could be applied, we required that it is fulfilled within $3 \sigma$. In addition we required that the $1 \sigma$ uncertainty in the spectral or temporal decay slopes was less than 0.2.

The final results of the light curve fits of the 27 bursts considered are presented in Fig. C.1. In addition, we plot here the observed flux-density ratio $\left(F_{v, \mathrm{opt}} / F_{v, \mathrm{x}}\right)(t)$ (middle panels in Fig. C.1) as a function of time. According to Table 1, the expected flux-density ratio is only allowed to take a certain value depending on the spectral and dynamical regime. We checked if the observed flux-density ratio, $\left(F_{v, \text { opt }} / F_{v, \mathrm{x}}\right)(t)$ (middle panels in Fig. C.1), agreed with the model(s) that successfully passed the previous three criteria. The allowed parameter space of the fluxdensity ratio of all considered models (Table 1) is shown as grey box in the middle panels in Fig. C.1. The upper and lower boundary always refer to $v_{\mathrm{c}} \leq v_{\mathrm{opt}}$ with $F_{v, \mathrm{opt}} / F_{v, \mathrm{x}}=\left(v_{\mathrm{opt}} / v_{\mathrm{x}}\right)^{-p / 2}$ and to $v_{\mathrm{c}} \geq v_{\mathrm{x}}$ with $F_{v, \mathrm{opt}} / F_{v, \mathrm{x}}=\left(v_{\mathrm{opt}} / v_{\mathrm{x}}\right)^{-(p-1) / 2}$. This criterion came into play when we were unable to distinguish whether the cooling frequency was redward of the optical band or blueward of the $\mathrm{X}$-ray band, in other words when the flux-density ratio agreed either with the lower or upper boundary in Fig. C.1.

\subsubsection{Ensemble properties}

Combining these different criteria, we found that for about $60 \%$ $(16 / 27)$ of all studied cases the cooling break was between the optical and the X-ray band (Table B.3), i.e., the difference in the decay slope is either $+1 / 4$ or $-1 / 4$ (for a constant density profile and a free wind medium, respectively). In total, we could identify the circumburst density profile (ISM or wind) for 25 of 
the 27 investigated afterglows (Table B.3). However, we identified a wind medium for only six events (GRBs, 050603, 070411, 080319B, 080514B, 080916C and 090323). The other 19 bursts were consistent with an ISM profile except for GRBs 051221A and 060904B.

Our procedure to find physical descriptions of light-curve segments, in other words identifying the spectral regime and the circumburst medium, allowed us to find descriptions of more optical and X-ray afterglows than are presented in the literature (Table B.3). Our results usually agree with the literature (for references see Table B.3); they only differ for GRBs 070802 , 080721, 090323, and 090328. The contradiction in the latter three bursts is due to the size of the optical data set. We used the maximum publicly available data set in contrast to Starling et al. (2009) (GRB 080721) and Cenko et al. (2010) (GRBs 090323 and 090328).

Four bursts are of particular interest in our sample:

(i) GRB 051221A ( $z=0.546$; Soderberg et al. 2006) is a short burst (Burrows et al. 2006), i.e., most likely it originated from the merger of two compact objects (Blinnikov et al. 1984; Paczyński 1986; Goodman 1986; Eichler et al. 1989). Unfortunately, we cannot discern between a wind or an ISM profile because $v_{\mathrm{c}}<v_{\mathrm{x}}$. The cooling frequency was below the optical bands so that neither the optical nor the X-ray data can be used to reveal the circumburst medium except during a post-jet break phase without lateral spreading.

(ii) GRB 060614 ( $z=0.125$; Della Valle et al. 2006b) is a quite controversial event that does not easily fit into the classical short/long classification scheme (Della Valle et al. 2006a; Fynbo et al. 2006; Gal-Yam et al. 2006; Gehrels et al. 2006; Mangano et al. 2007; Zhang et al. 2007; Kann et al. 2008; Zhang et al. 2009). The difference in the spectral slope, $\beta_{\mathrm{x}}-\beta_{\mathrm{opt}}=0.00 \pm 0.11$ (Table B.3), rules out that the cooling frequency lies between the optical and $\mathrm{X}$-ray bands. Furthermore, we did not find evidence for a wind medium. The optical and X-ray data exclude a wind medium with high confidence. The deviation between the observed and predicted temporal decay slope is $<1.3 \sigma$ and $>3.5 \sigma$ for an ISM and a wind medium, respectively. In our sample this burst belongs to a small number of cases where the flux density ratio could be used to distinguish between $v_{\mathrm{c}}>\left(v_{\mathrm{opt}}, v_{\mathrm{x}}\right)$ and $v_{\mathrm{c}}<\left(v_{\mathrm{opt}}, v_{\mathrm{x}}\right)$. The small observed flux density ratio agrees with $v_{\mathrm{c}}>\left(v_{\mathrm{opt}}, v_{\mathrm{x}}\right)$.

(iii) GRB 060904B has a well-defined light curve (Fig. C.1, Table B.2) and SED (Table B.1) in the optical and X-ray bands, respectively. The difference in the decay slopes, $\alpha_{\mathrm{x}}-\alpha_{\text {opt }}=0.20 \pm 0.04$ (Table B.3), favours an ISM profile with the cooling frequency lying between the optical and the X-ray bands. The difference in the spectral slopes, however, does not support this scenario, $\beta_{\mathrm{x}}-\beta_{\text {opt }}=0.00 \pm 0.16$ (Table B.3). Rather, both afterglow components seem to be in the same spectral regime. Therefore, we could not find a consistent description of the optical and X-ray afterglow. The main reason could be that the optical data are mainly based on preliminary data (see Kann et al. 2010 for details on the data gathering).

(iv) GRB 080319B ( $z=0.937$; D'Elia et al. 2009) is the only burst in our sample with a photometrically detected supernova component (Bloom et al. 2009; Tanvir et al. 2008). An ISM profile can be ruled out with very high confidence. The difference in the spectral slopes, $\beta_{\mathrm{x}}-\beta_{\text {opt }}=0.48 \pm 0.12$ (Table B.3), favours the cooling break to be in between the optical and the X-ray bands. The deviation between
$\Delta \alpha_{\mathrm{obs}}=\alpha_{\mathrm{x}}-\alpha_{\mathrm{opt}}$ and $\Delta \alpha_{\text {predicted }}$ is $1 \sigma$ for a wind medium but $9 \sigma$ for an ISM profile. The closure relations support this finding. The deviation between the observed and predicted optical decay slope is $0.1 \sigma$ for a wind medium and $4.6 \sigma$ for an ISM profile (see also Racusin et al. 2008).

\subsubsection{The electron index}

Finally, the identification of the light-curve segments allowed us to derive the electron index, $p$. It is shown in the top panels in Fig. C.1 for every burst. In agreement with other studies (e.g., Panaitescu \& Kumar 2001; Shen et al. 2006; Zeh et al. 2006; Starling et al. 2008; Curran et al. 2009; Ghisellini et al. 2009; Curran et al. 2010) we find that the distribution extends from $p \sim 2$ to $p \sim 3$, indicating that $p$ is no universal value.

\subsection{Constraining the wind - ISM transition zone}

Once we had identified the circumburst density profiles, we could investigate if there is observational evidence for the position of the wind termination-shock radius, where the density profile changes from that of a free wind $(k=2)$ to a density profile with $k=0$ (independent of whether it is the shocked wind or the ISM; see Pe'er \& Wijers 2006; van Marle et al. 2007). While the theory of a blastwave crossing such a density discontinuity has been worked out (Pe'er \& Wijers 2006), finding the corresponding observational signature is difficult. Even though data on several hundred afterglows exist, they do not provide this kind of information in a convincing way (Starling et al. 2008; Curran et al. 2009). This leaves open the question of observationally determining the wind termination-shock radius.

Here we cannot determine the radius of the wind termination shock for any (long) GRB progenitor either. However, we can characterise its position in a statistical sense. The light curves in Fig. C.1 show $F_{\text {opt }} / F_{\mathrm{x}}$ as a function of time. Its first logarithmic derivative, $\left(\alpha_{\mathrm{x}}-\alpha_{\mathrm{opt}}\right)(t)$, reveals either the maximum time up to which a wind profile is identified or it reveals the minimum time after which an ISM profile agrees with the data. The function $\left(\alpha_{\mathrm{x}}-\alpha_{\text {opt }}\right)(t)$ is a smooth function in time in contrast to the fit values, because we used a smoothly broken power law to describe the light-curve evolution. Table 3 summarises the time intervals for which the asymptotic values for $\alpha_{\mathrm{x}}-\alpha_{\mathrm{opt}}$ were reached, i.e. $-1 / 4,0$, or $1 / 4$ within $1 \sigma$.

In the observer frame, the radius of the fireball propagating into a free wind medium is (Chevalier \& Li 2000)

$R(t)=1.1 \times 10^{17}\left(\frac{2 t E_{\mathrm{iso}, 52}}{(1+z) A_{\star}}\right)^{1 / 2} \mathrm{~cm}$,

where $t$ is measured in units of days, $E_{\text {iso }}$ is the isotropic equivalent energy in units of $10^{52} \mathrm{erg}$ and $A_{\star}$ is defined via $A=$ $\dot{M}_{\mathrm{w}} / 4 \pi v_{\mathrm{w}}=5 \times 10^{11} A_{\star} \mathrm{g} \mathrm{cm}^{-1}$, with $\dot{M}_{\mathrm{w}}$ being the mass-loss rate, and $v_{\mathrm{w}}$ the wind velocity. The quantity $A_{\star}$ refers to a massloss rate of $\dot{M}_{\mathrm{w}}=10^{-5} M_{\odot} \mathrm{yr}^{-1}$ and a velocity of the stellar wind of $v_{\mathrm{w}}=10^{8} \mathrm{~cm} \mathrm{~s}^{-1}$.

Using the aforementioned approach and fixing for simplicity $A_{\star}=1$, Table 3 summarises the deduced upper and lower limits on the wind termination-shock radius. The distribution extends over three orders of magnitude (from $\approx 10^{-3} \mathrm{pc}$ to $1 \mathrm{pc}$ ). This spread is partly due to the strong correlation with $E_{\text {iso }}(R(t) \propto$ $\left.E_{\text {iso }}^{1 / 2}\right)$ and is thus related to the spread in the energy released during the prompt emission in gamma-rays (width $\approx 4.3 \mathrm{dex}$ ). On the other hand, since $R(t)$ scales with $A_{\star}^{-1 / 2}, A_{\star}$ would have to 
Table 3. Constraints on the termination-shock radii.

\begin{tabular}{|c|c|c|c|c|}
\hline GRB & $\begin{array}{l}\tilde{t}_{\text {start }} \\
(\mathrm{ks}) \\
\end{array}$ & $\begin{array}{r}\tilde{t}_{\text {end }} \\
(\mathrm{ks}) \\
\end{array}$ & $\begin{array}{c}\log E_{\text {iso }} \\
\text { [erg] }\end{array}$ & $\begin{array}{c}R_{T} \\
(\mathrm{pc}) \\
\end{array}$ \\
\hline \multicolumn{5}{|c|}{ Constant density medium $\left(n(r) \propto r^{0}\right)$} \\
\hline 050801 & 0.31 & 105.70 & $51.51_{-0.12}^{+0.34}$ & $<0.0011_{-0.0001}^{+0.0005}$ \\
\hline 050820A & 42.71 & 411.00 & $53.99_{-0.06}^{+0.11}$ & $<0.18_{-0.01}^{+0.02}$ \\
\hline 051109A & 25.87 & 264.60 & $52.87_{-0.89}^{+0.08}$ & $<0.04_{-0.03}^{+0.01}$ \\
\hline 060418 & 4.40 & 535.80 & $53.15_{-0.10}^{+0.13}$ & $<0.027_{-0.003}^{+0.004}$ \\
\hline 060512 & 10.50 & 287.50 & $50.30_{-0.10}^{+0.40}$ & $<0.0021_{-0.0002}^{+0.0012}$ \\
\hline 060614 & 51.59 & 1497.00 & $51.40_{-0.04}^{+0.05}$ & $<0.018 \pm 0.001$ \\
\hline 060714 & 78.49 & 286.10 & $52.89_{-0.05}^{+0.30}$ & $<0.069_{-0.004}^{+0.029}$ \\
\hline 060908 & 0.92 & 82.14 & $52.82 \pm 0.20$ & $<0.008 \pm 0.002$ \\
\hline 070802 & 10.90 & 88.78 & $51.70_{-0.09}^{+0.31}$ & $<0.007_{-0.001}^{+0.003}$ \\
\hline 070810A & 1.95 & 34.41 & $51.96_{-0.16}^{+0.05}$ & $<0.0041_{-0.0007}^{+0.0002}$ \\
\hline 080710 & 23.10 & 348.50 & $51.90_{-0.32}^{+0.31}$ & $<0.02 \pm 0.01$ \\
\hline 080721 & 29.46 & 1258.00 & $54.09_{-0.04}^{+0.03}$ & $<0.17 \pm 0.01$ \\
\hline 081203A & 12.07 & 301.00 & $53.54_{-0.15}^{+0.18}$ & $<0.06 \pm 0.01$ \\
\hline 090102 & 77.57 & 262.80 & $53.30 \pm 0.04$ & $<0.13 \pm 0.01$ \\
\hline 090328 & 57.89 & 923.30 & $52.99 \pm 0.01$ & $<0.098 \pm 0.001$ \\
\hline 090726 & 3.61 & 10.00 & $52.26_{-0.10}^{+0.33}$ & $<0.007_{-0.001}^{+0.003}$ \\
\hline 090902B & 45.64 & 1168.00 & $54.49 \pm 0.01$ & $<0.383 \pm 0.004$ \\
\hline 090926A & 325.80 & 1798.00 & $54.27 \pm 0.02$ & $<0.76 \pm 0.02$ \\
\hline \multicolumn{5}{|c|}{ Free stellar wind $\left(n(r) \propto r^{-2}\right)$} \\
\hline 050603 & 39.21 & 196.50 & $53.79 \pm 0.01$ & $>0.305 \pm 0.004$ \\
\hline 070411 & 92.09 & 522.70 & $53.00_{-0.10}^{+0.26}$ & $>0.20_{-0.02}^{+0.07}$ \\
\hline 080319B & 52.76 & 555.70 & $54.16 \pm 0.01$ & $>1.11 \pm 0.01$ \\
\hline 080514B & 37.30 & 174.70 & $53.42_{-0.04}^{+0.03}$ & $>0.22 \pm 0.01$ \\
\hline 080916C & 97.49 & 377.70 & $54.49_{-0.10}^{+0.08}$ & $>0.80_{-0.09}^{+0.08}$ \\
\hline 090323 & 73.05 & 1079.00 & $54.61 \pm 0.01$ & $>1.68 \pm 0.02$ \\
\hline \multicolumn{5}{|c|}{ Unclear cases } \\
\hline 050922C & $\ldots$ & & $52.98_{-0.05}^{+0.08}$ & $\ldots$ \\
\hline 051221A & 25.92 & 228.80 & $51.41_{-0.33}^{+0.02}$ & $\ldots$ \\
\hline 060904B & 3.58 & 162.80 & $51.71_{-0.21}^{+0.13}$ & $\ldots$ \\
\hline
\end{tabular}

Notes. Time intervals deduced from the lower panels in Fig. C.1 if the first logarithmic derivative of $F_{\text {opt }} / F_{\mathrm{x}}$ points to either an ISM profile or a free wind, i.e., once the asymptotic value $+1 / 4$ or $-1 / 4$ was reached (within the error bars). With the exception for GRB 080514B (Rossi et al. 2009) and GRB 090726 (Butler et al. 2010), the isotropic equivalent energies, $E_{\text {iso }}$, were taken from Kann et al. $(2008,2010)$. The forth column was calculated based on Eq. (1), assuming $A_{*}=1$, with $t=\tilde{t}_{\text {start }}$ (Fig. C.1) for ISM profiles and $t=\tilde{t}_{\text {end }}$ (Fig. C.1) for wind profiles.

vary by a factor of 100 in the right way to reduce the width of this distribution by only a factor of 10 .

Is the width of the distribution we have found for the upper limits on the wind termination shock reasonable? Based on numerical wind models of Wolf-Rayet stars, Fryer et al. (2006) found that for $A_{\star}=1$ the radius of the termination shock is approximately given by

$R_{\mathrm{T}}=\left(n_{\mathrm{ISM}} / 100 \mathrm{~cm}^{-3}\right)^{-1 / 2} \mathrm{pc}$.

ISM densities of the order of $10^{6} \mathrm{~cm}^{-3}$ are then required to reduce $R_{\mathrm{T}}$ to $0.01 \mathrm{pc}$. These large-scale gas densities are rather unique and only typical for dense cores of molecular clouds (with the Rho Ophiuchi Cloud as an example, e.g., Klose 1986).

Observationally, gas densities could only be derived for a few bursts because of the lack of radio data. So far, the highest values are about $600 \mathrm{~cm}^{-3}$ (Frail et al. 2006; Thöne et al. 2010),

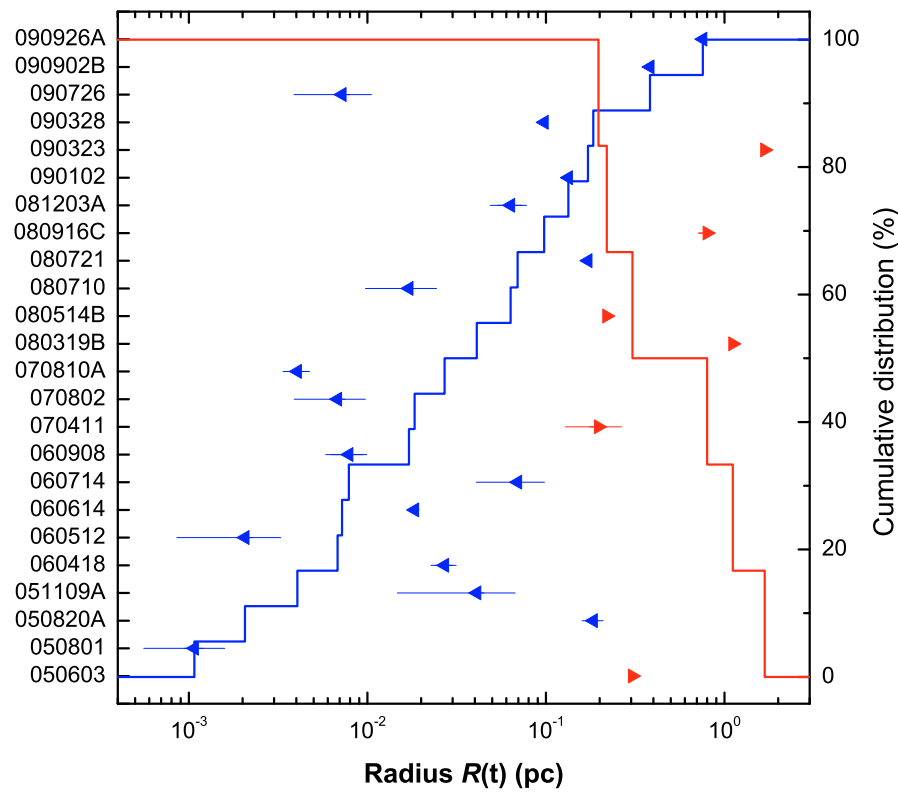

Fig. 1. Shown here are lower (to-the-right pointing triangles) and upper (to-the-left pointing triangles) limits on the position of the wind termination shock based on Eq. (1), assuming $A_{\star}=1$ in all cases (Table 3 ). Note that GRB 060614 is a much debated burst (see Sect. 3.1.1). The step curves are the cumulative distributions to the lower limits up to which a wind profile is identified and upper limits after which a constant density medium (ISM) agrees with the data.

while the nominal value is about a factor of 100 smaller (Frail et al. 2006). These measurements do not necessarily rule out the model of Fryer et al. (2006). Successful radio observations might have picked out a certain class of GRBs. Furthermore, radio observations are very challenging at early times because the brightness of the afterglow is increasing in the radio bands while the afterglow is already decaying in the optical and X-ray bands (Zhang \& Mészáros 2004). Thus, it is difficult to extract information on the direct vicinity of the progenitor. On the other hand, if the average particle density of the circumburst medium is about $1-10 \mathrm{~cm}^{-3}$, then additional mechanisms are required to bring the wind termination-shock radius closer to the star (e.g., van Marle et al. 2006).

The other possibility to reduce $R_{\mathrm{T}}$ is to decrease $A_{\star}$, since $R_{\mathrm{T}} \propto A_{\star}^{1 / 2}$ (Chevalier et al. 2004). For example, for $A_{\star}=0.01$ all data points in Fig. 1 would shift along the $R$-coordinate to higher values by a factor of 10 (Eq. (1)), while $R_{\mathrm{T}}$ would decrease by the same factor. In this case, lower circumburst gas densities would be required. This touches upon the question on how small $A_{\star}$ can be. Studies of WR stars do not favour values of less than 0.01 in polar directions (Eldridge 2007, his Table 1). Moreover, observations of nearby WR stars do not show evidence for these low values either (Nugis \& Lamers 2000). However, the majority of nearby WR stars are surely not seen pole-on, in contrast to GRBs. Therefore, it is difficult to decide if these observational constraints on WR stars can be applied to GRB progenitors.

On the other hand, the six GRBs that favour a free wind medium (Table B.3) have large lower limits on the wind termination-shock radius (Table 3 ). This matches theoretical models by van Marle et al. $(2007,2008)$, which allow $R_{\mathrm{T}}$ to extend up to several parsecs.

The separation between the lower and upper limits for wind and ISM-profiles, respectively, on the wind termination-shock 
radius could be even larger. Refining the lower and upper limits is difficult, however. The lower limits, which are deduced from $\tilde{t}_{\text {end }}$ (Fig. C.1, Table 3), depend on the observing strategies due to the brightness of the afterglow and the brightness of the underlying host galaxy in the optical bands. On the other hand, the upper limits, which are deduced from $\tilde{t}_{\text {start }}$ (Fig. C.1, Table 3), can be affected by additional radiation components at early times.

\section{Summary and conclusion}

After applying several selection criteria (closure relations, the differences in the spectral and temporal slopes, and the flux density ratio, $F_{\text {opt }} / F_{\mathrm{x}}$ ) we selected the best-sampled Swift GRBs with well-observed optical as well as X-ray afterglow data from June 2005 to September 2009. Altogether 27 bursts entered our sample, which was used to investigate the density profile of the circumburst medium (constant density medium or free wind), including one short burst (GRB 051221A) and one controversial event in terms of classification (GRB 060614), which successfully passed our selection criteria among all bursts. The other 25 events are classified as long bursts without doubt.

Combining optical with X-ray data is advantageous because optical data usually allow for a more precise determination of the temporal decay slope of an afterglow, while X-ray data can in general be used to extract the SED. Combining both emission components substantially improves our capability to distinguish between an ISM and a wind medium. Thereby, we concentrated on the late-time evolution, i.e., times when the proper afterglow is not affected anymore by flares and additional radiation components (e.g., the reverse shock, central engine activity).

Our study shows that only six of the 25 long bursts (24\%) investigated here (GRBs 050603, 070411, 080319B, 080514B, 080916C and 090323) showed evidence for a free wind medium at late times. In the other cases (76\%), except for the short burst GRB 051221A and 060904B, the blastwaves were propagating into a constant density-medium. In particular, the controversial burst GRB 060614 favours an ISM profile. This is not in disagreement with a massive-star origin as our result for long bursts indicates.

In addition, we were able to set limits on the wind termination-shock radii of the corresponding GRB progenitors. Only 24 of 27 bursts (Table 3) had good enough data to perform this analysis. Fixing the relative mass-loss rate to $A_{\star}=1$, the distribution we deduced covers three orders of magnitude. We find a tentative grouping into (long) GRB progenitors with comparably small and comparably large termination-shock radii. Whether this points to two distinct populations of (long) GRB progenitors or if this is a selection effect, remains an open issue. At least theoretically it is well possible that the long burst population splits into single star progenitors and those belonging to a binary system (Georgy et al. 2009). Further observational data are required to reveal a potential binary nature of the long burst progenitors.

Acknowledgements. We thank the referee for a very careful reading of the manuscript and a rapid reply. S.S. acknowledges support by a Grant of Excellence from the Icelandic Research Fund and Thüringer Landessternwarte Tautenburg, Germany, where part of this study was performed. D.A.K. acknowledges support from grant DFG Kl 766/16-1. A.R. acknowledges support from the BLANCEFLOR Boncompagni-Ludovisi, née Bildt foundation. T.K. acknowledges support by the DFG cluster of excellence "Origin and Structure of the Universe". S.S. acknowledges Robert Chapman (U Iceland), Elisabetta Maiorano (CNR Bologna), Andrea Mehner (U Minnesota), Kim Page (U Leicester), Eliana Palazzi (CNR Bologna) and Gunnar Stefansson (U Iceland) for helpful discussions. This work made use of data supplied by the UK Swift Science Data Centre at the University of Leicester.

\section{References}

Berger, E., \& Becker, G. 2005, GCN Circ., 3520

Berger, E., Penprase, B. E., Cenko, S. B., et al. 2006, ApJ, 642, 979

Bessell, M. S. 1979, PASP, 91, 589

Beuermann, K., Hessman, F. V., Reinsch, K., et al. 1999, A\&A, 352, L26

Blinnikov, S. I., Novikov, I. D., Perevodchikova, T. V., \& Polnarev, A. G. 1984 SvA Lett., 10, 177

Bloom, J. S., Foley, R. J., Koceveki, D., \& Perley, D. 2006, GCN Circ., 5217

Bloom, J. S., Perley, D. A., Li, W., et al. 2009, ApJ, 691, 723

Burrows, D. N., Grupe, D., Capalbi, M., et al. 2006, ApJ, 653, 468

Butler, N. R., Bloom, J. S., \& Poznanski, D. 2010, ApJ, 711, 495

Castro-Tirado, A. J., Møller, P., García-Segura, G., et al. 2010, A\&A, 517, A61

Cenko, S. B., Frail, D. A., Harrison, F. A., et al. 2010, ApJ, submitted [arXiv: 1004.2900$]$

Cenko, S. B., Kasliwal, M., Harrison, F. A., et al. 2006, ApJ, 652, 490

Chen, H., Prochaska, J. X., Ramirez-Ruiz, E., et al. 2007, ApJ, 663, 420

Chevalier, R. A., \& Li, Z.-Y. 1999, ApJ, 520, L29

Chevalier, R. A., \& Li, Z.-Y. 2000, ApJ, 536, 195

Chevalier, R. A., Li, Z.-Y., \& Fransson, C. 2004, ApJ, 606, 369

Chornock, R., Berger, E., Levesque, E. M., et al. 2010, ApJ, submitted [arXiv: 1004.2262]

Costa, E., Frontera, F., Heise, J., et al. 1997, Nature, 387, 783

Crowther, P. A. 2007, ARA\&A, 45, 177

Curran, P. A., Evans, P. A., de Pasquale, M., Page, M. J., \& van der Horst, A. J. 2010, ApJ, 716, L135

Curran, P. A., Starling, R. L. C., van der Horst, A. J., \& Wijers, R. A. M. J. 2009, MNRAS, 395, 580

Dado, S., Dar, A., \& De Rújula, A. 2002, A\&A, 388, 1079

de Pasquale, M., Oates, S. R., Page, M. J., et al. 2007, MNRAS, 377, 1638

de Ugarte Postigo, A., Jakobsson, P., Malesani, D., et al. 2009, GCN Circ., 8766

D’Elia, V., Fiore, F., Perna, R., et al. 2009, ApJ, 694, 332

Della Valle, M., Chincarini, G., Panagia, N., et al. 2006a, Nature, 444, 1050

Della Valle, M., Malesani, D., Bloom, J. S., et al. 2006b, ApJ, 642, L103

Della Valle, M., Benetti, S., Mazzali, P., et al. 2008, Central Bureau Electronic Telegrams, 1602

Eichler, D., Livio, M., Piran, T., \& Schramm, D. N. 1989, Nature, 340, 126 Eldridge, J. J. 2007, MNRAS, 377, L29

Evans, P. A., Beardmore, A. P., Page, K. L., et al. 2007, A\&A, 469, 379

Evans, P. A., Beardmore, A. P., Page, K. L., et al. 2009, MNRAS, 397, 1177

Fatkhullin , T., Gorosabel, J., de Ugarte Postigo, A., et al. 2009, GCN Circ., 9712

Ferrero, P., Kann, D. A., Zeh, A., et al. 2006, A\&A, 457, 857

Filippenko, A. V. 1997, ARA\&A, 35, 309

Fox, A. J., Ledoux, C., Vreeswijk, P. M., Smette, A., \& Jaunsen, A. O. 2008, A\&A, 491, 189

Frail, D. A., Cameron, P. B., Kasliwal, M., et al. 2006, ApJ, 646, L99

Fryer, C. L., Rockefeller, G., \& Young, P. A. 2006, ApJ, 647, 1269

Fynbo, J. P. U., Watson, D., Thöne, C. C., et al. 2006, Nature, 444, 1047

Fynbo, J. P. U., Jakobsson, P., Prochaska, J. X., et al. 2009, ApJS, 185, 526

Gal-Yam, A., Fox, D. B., Price, P. A., et al. 2006, Nature, 444, 1053

Galama, T. J., Vreeswijk, P. M., van Paradijs, J., et al. 1998, Nature, 395, 670

Galama, T. J., Tanvir, N., Vreeswijk, P. M., et al. 2000, ApJ, 536, 185

Gehrels, N., Norris, J. P., Barthelmy, S. D., et al. 2006, Nature, 444, 1044

Gehrels, N., Barthelmy, S. D., Burrows, D. N., et al. 2008, ApJ, 689, 1161

Gendre, B., Klotz, A., Palazzi, E., et al. 2010, MNRAS, 405, 2372

Georgy, C., Meynet, G., Walder, R., Folini, D., \& Maeder, A. 2009, A\&A, 502, 611

Ghisellini, G., Nardini, M., Ghirlanda, G., \& Celotti, A. 2009, MNRAS, 393, 253

Goodman, J. 1986, ApJ, 308, L47

Greiner, J., Clemens, C., Krühler, T., et al. 2009a, A\&A, 498, 89

Greiner, J., Krühler, T., Fynbo, J. P. U., et al. 2009b, ApJ, 693, 1610

Haislip, J., Reichart, D., Cominsky, L., McLin, K., et al. 2009, GCN Circ., 9926

Hjorth, J., Sollerman, J., Møller, P., et al. 2003, Nature, 423, 847

Iwamoto, K., Mazzali, P. A., Nomoto, K., et al. 1998, Nature, 395, 672

Jakobsson, P., Fynbo, J. P. U., Ledoux, C., et al. 2006, A\&A, 460, L13

Kalberla, P. M. W., Burton, W. B., Hartmann, D., et al. 2005, A\&A, 440, 775

Kann, D. A., Klose, S., \& Zeh, A. 2006, ApJ, 641, 993

Kann, D. A., Klose, S., Zhang, B., et al. 2008, ApJ, submitted [arXiv: 0804.1959]

Kann, D. A., Klose, S., Zhang, B., et al. 2010, ApJ, 720, 1513

Kawabata, K. S., Deng, J., Wang, L., et al. 2003, ApJ, 593, L19

Kelemen, J. 2009, GCN Circ., 10028

Klose, S. 1986, Ap\&SS, 128, 135

Klose, S., Greiner, J., Rau, A., et al. 2004, AJ, 128, 1942

Krühler, T., Küpcü Yoldaș, A., Greiner, J., et al. 2008, ApJ, 685, 376

Krühler, T., Greiner, J., Afonso, P., et al. 2009, A\&A, 508, 593

Kuin, N. P. M., Landsman, W. B., Page, M. J., et al. 2009, MNRAS, 395, L21 
Landsman, W. B. \& Page, K. 2009, GCN Circ., 9717

Liang, E., Racusin, J. L., Zhang, B., Zhang, B., \& Burrows, D. N. 2008, ApJ, 675,528

Malesani, D., Tagliaferri, G., Chincarini, G., et al. 2004, ApJ, 609, L5

Mangano, V., Holland, S. T., Malesani, D., et al. 2007, A\&A, 470, 105

Masetti, N., Palazzi, E., Pian, E., et al. 2005, A\&A, 438, 841

Matheson, T., Garnavich, P. M., Stanek, K. Z., et al. 2003, ApJ, 599, 394

McBreen, S., Krühler, T., Rau, A., et al. 2010, A\&A, 516, 71

Mirabal, N., Halpern, J. P., An, D., Thorstensen, J. R., \& Terndrup, D. M. 2006, ApJ, 643, L99

Mirabal, N., Halpern, J. P., Chornock, R., et al. 2003, ApJ, 595, 935

Modjaz, M., Stanek, K. Z., Garnavich, P. M., et al. 2006, ApJ, 645, L21

Moretti, A., Campana, S., Mineo, T., et al. 2005, in Presented at the Society of Photo-Optical Instrumentation Engineers (SPIE) Conference, UV, X-Ray, and Gamma-Ray Space Instrumentation for Astronomy XIV, ed. O. H. W. Siegmund, Proc. SPIE, 5898, 360

Nousek, J. A., Kouveliotou, C., Grupe, D., et al. 2006, ApJ, 642, 389

Nugis, T., \& Lamers, H. J. G. L. M. 2000, A\&A, 360, 227

Paczyński, B. 1986, ApJ, 308, L43

Panaitescu, A. 2007, MNRAS, 380, 374

Panaitescu, A., \& Kumar, P. 2001, ApJ, 560, L49

Panaitescu, A., \& Vestrand, W. T. 2010, MNRAS, submitted [arXiv: 1009.3947]

Pe'er, A., \& Wijers, R. A. M. J. 2006, ApJ, 643, 1036

Pian, E., Mazzali, P. A., Masetti, N., et al. 2006, Nature, 442, 1011

Piranomonte, S., Ward, P. A., Fiore, F., et al. 2008, A\&A, 492, 775

Press, W. H., Teukolsky, S. A., Vetterling, \& W. T., Flannery, B. P. 2007 Numerical Recipes, 3rd edn. (Cambridge University Press)

Prochaska, J. X., Chen, H. W., Bloom, J. S., Falco, E., \& Dupree, A. K. 2006, GCN Circ., 5002

Prochaska, J. X., Chen, H., Dessauges-Zavadsky, M., \& Bloom, J. S. 2007, ApJ, 666, 267

Quimby, R., Fox, D., Hoeflich, P., Roman, B., \& Wheeler, J. C. 2005, GCN Circ., 4221

Racusin, J. L., Karpov, S. V., Sokolowski, M., et al. 2008, Nature, 455, 183

Rau, A., Savaglio, S., Krühler, T., et al. 2010, ApJ, 720, 862

Reichart, D. E. 1999, ApJ, 521, L111

Romano, P., Campana, S., Chincarini, G., et al. 2006, A\&A, 456, 917

Rossi, A., de Ugarte Postigo, A., Ferrero, P., et al. 2009, A\&A, 491, L29
Rykoff, E. S., Mangano, V., Yost, S. A., et al. 2006, ApJ, 638, L5

Sakamoto, T., Donato, D., Gehrels, N., et al. 2009, GCN Circ., 9732

Sari, R., Piran, T., \& Narayan, R. 1998, ApJ, 497, L17

Schady, P., Mason, K. O., Page, M. J., et al. 2007, MNRAS, 377, 273

Schady, P., Page, M. J., Oates, S. R., et al. 2010, MNRAS, 401, 2773

Schaefer, B. E., Gerardy, C. L., Höflich, P., et al. 2003, ApJ, 588, 387

Shen, R., Kumar, P., \& Robinson, E. L. 2006, MNRAS, 371, 1441

Soderberg, A. M., Kulkarni, S. R., Price, P. A., et al. 2006, ApJ, 636, 391

Sollerman, J., Kozma, C., Fransson, C., et al. 2000, ApJ, 537, L127

Sollerman, J., Jaunsen, A. O., Fynbo, J. P. U., et al. 2006, A\&A, 454, 503

Stanek, K. Z., Matheson, T., Garnavich, P. M., et al. 2003, ApJ, 591, L17

Starling, R. L. C., Wijers, R. A. M. J., Hughes, M. A., et al. 2005, MNRAS, 360, 305

Starling, R. L. C., van der Horst, A. J., Rol, E., et al. 2008, ApJ, 672, 433

Starling, R. L. C., Rol, E., van der Horst, A. J., et al. 2009, MNRAS, 400, 90

Starling, R. L. C., Wiersema, K., Levan, A. J., et al. 2010, MNRAS, in press [arXiv: $1004.2919 \mathrm{v} 2]$

Tanvir, N. R., Rol, E., Levan, A., et al. 2008, ApJ, 725, 625

Thöne, C. C., Perley, D. A., Cooke, J., et al. 2007, GCN Circ., 6741

Thöne, C. C., Kann, D. A., Jóhannesson, G., et al. 2010, A\&A, 523, A70

Šimon, V., Polášek, C., Jelínek, M., Hudec, R., \& Trobl, J. Å. 2010, A\&A, 510, A49

van Marle, A. J., Langer, N., Achterberg, A., \& García-Segura, G. 2006, A\&A 460,105

van Marle, A. J., Langer, N., \& García-Segura, G. 2007, A\&A, 469, 941

van Marle, A. J., Langer, N., Yoon, S., \& García-Segura, G. 2008, A\&A, 478, 769

van Paradijs, J., Groot, P. J., Galama, T. J., et al. 1997, Nature, 386, 686

Vaughan, S., Goad, M. R., Beardmore, A. P., et al. 2006, ApJ, 638, 920

Volnova, A., Pavlenko, E., Sklyanov, A., Antoniuk, O., \& Pozanenko, A. 2009 GCN Circ., 9741

Wilms, J., Allen, A., \& McCray, R. 2000, ApJ, 542, 914

Woosley, S. E., \& Bloom, J. S. 2006, ARA\&A, 44, 507

Zeh, A., Klose, S., \& Hartmann, D. H. 2004, ApJ, 609, 952

Zeh, A., Klose, S., \& Kann, D. A. 2006, ApJ, 637, 889

Zhang, B., \& Mészáros, P. 2004, Int. J. Mod. Phys. A, 19, 2385

Zhang, B., Fan, Y. Z., Dyks, J., et al. 2006, ApJ, 642, 354

Zhang, B., Zhang, B.-B., Liang, E.-W., et al. 2007, ApJ, 655, L25

Zhang, B., Zhang, B., Virgili, F. J., et al. 2009, ApJ, 703, 1696 


\section{Appendix A: Smoothly broken power law of the order $m$}

The equation for a smoothly broken power-law of the order $m$, $F_{v, m}(t)$, was derived by recursion in the following way. Let us assume the function $F_{v, m}(t)$ consists of $m$ power-law segments connected by $(m-1)$ breaks. To add an additional power-law segment $\tilde{F}_{v, m+1}(t)$, we first normalised the new power-law segment to the previous one, $F_{v, m}(t)$, at the break time $t_{b, m}$ :

$\tilde{F}_{v, m+1}(t)=F_{v, m}\left(t_{b, m}\right)\left(\frac{t}{t_{b, m}}\right)^{-\alpha_{m+1}}$.

Here $\alpha_{m+1}$ is the slope of segment $(m+1)$. Second, we followed Beuermann et al. (1999) and introduced a smoothness parameter $n_{m}$ so that the smoothly broken power law of order the $(m+1)$ takes the form

$F_{v, m+1}(t)=\left(F_{v, m}^{-n_{m}}(t)+\tilde{F}_{v, m+1}^{-n_{m}}(t)\right)^{-1 / n_{m}}$.

If the light-curve consists of $m$ segments, both steps (adding and smoothing) have to be performed $(m-1)$-times.

For example, let us derive the equation for a smoothly broken power-law (Beuermann et al. 1999). In this case $m=2$, thus the function consists of two power-law segments connected by one break at the time $t_{b, 1}$. The initial function is a simple power law $F_{v, 1}(t)=C t^{-\alpha_{1}}$. First, the second power-law segment, $\tilde{F}_{v, 2}$, has to be connected to the first one at the time $t_{b, 1}$ ( $\operatorname{step}$ A.1)

$$
\begin{aligned}
\tilde{F}_{v, 2} & =F_{v, 1}\left(t_{b, 1}\right)\left(\frac{t}{t_{b, 1}}\right)^{-\alpha_{2}} \\
& =C t_{b, 1}^{-\alpha_{1}}\left(\frac{t}{t_{b, 1}}\right)^{-\alpha_{2}} .
\end{aligned}
$$

Second, the transition has to be smoothed by weighting both functions at the point of intersection (step A.2)

$$
\begin{aligned}
F_{v, 2} & =\left(F_{v, 1}^{-n_{1}}(t)+\tilde{F}_{v, 2}^{-n_{1}}(t)\right)^{-1 / n_{1}} \\
& =\left(C^{-n_{1}} t^{\alpha_{1} n_{1}}+C^{-n_{1}} t_{b, 1}^{\alpha_{1} n_{1}}\left(\frac{t}{t_{b, 1}}\right)^{\alpha_{2} n_{1}}\right)^{-1 / n_{1}} \\
& =C t_{b, 1}^{-\alpha_{1}}\left(\left(\frac{t}{t_{b, 1}}\right)^{\alpha_{1} n_{1}}+\left(\frac{t}{t_{b, 1}}\right)^{\alpha_{2} n_{1}}\right)^{-1 / n_{1}} .
\end{aligned}
$$

This leads to the equation found by Beuermann et al. (1999) for a smoothly broken power-law. Repeating both steps leads to a smoothly broken power-law of the order 3 (double smoothly broken power-law; Liang et al. 2008). Thus, looping ( $m-1)$-times over both steps results in a smoothly broken power law of the order $m$. 
S. Schulze et al.: The circumburst density profile around GRB progenitors: a statistical study

\section{Appendix B: Tables}

Table B.1. Properties of the afterglow SEDs in the optical and X-ray bands of the 27 bursts that entered our sample.

\begin{tabular}{|c|c|c|c|c|c|c|c|}
\hline GRB & $z$ & $\beta_{\mathrm{opt}}$ & $\begin{array}{c}\text { Dust } \\
\text { Model }\end{array}$ & $A_{V}^{\text {host }}$ & $\beta_{\mathrm{x}}$ & $\begin{array}{l}t_{\mathrm{mid}} \\
(\mathrm{ks})\end{array}$ & References \\
\hline 050603 & 2.818 & & & & $0.96 \pm 0.12$ & $\mathrm{X}: 44$ & $z: 1 ;$ Opt: 2 \\
\hline 050801 & 1.560 & $0.69 \pm 0.34$ & SMC & $0.30 \pm 0.18$ & $0.89 \pm 0.08$ & Opt: 86; X: 79 & $z: 3 ;$ Opt: 2 \\
\hline 050820A & 2.615 & $0.72 \pm 0.03$ & SMC & $0.07 \pm 0.01$ & $1.09 \pm 0.03$ & Opt: 86; X: 37 & $z: 4 ;$ Opt: 2 \\
\hline $050922 \mathrm{C} \dagger$ & 2.199 & 1.07 & MW & $0.17 \pm 0.05$ & $1.07 \pm 0.05$ & Opt/X: 20 & $z: 6 ; \mathrm{Opt} / \mathrm{X}: 5$ \\
\hline 051109A $\dagger$ & 2.346 & 0.40 & SMC & $<0.10$ & $0.90 \pm 0.04$ & Opt/X: 5 & $z: 7 ; \mathrm{Opt} / \mathrm{X}: 5$ \\
\hline $051221 \mathrm{~A}$ & 0.546 & $\ldots$ & & $\ldots$ & $0.95 \pm 0.11$ & $\mathrm{X}: 3$ & $z: 8 ;$ Opt: 9 \\
\hline 060418 & 1.490 & $0.69 \pm 0.11$ & LMC & $0.20 \pm 0.08$ & $0.98 \pm 0.15$ & Opt: $86 ; \mathrm{X}: 7$ & $z: 10 ;$ Opt: 2 \\
\hline 060512 & 0.443 & $\ldots$ & & & $1.02 \pm 0.11$ & $X: 23$ & $z: 11$ \\
\hline 060614 & 0.125 & $0.81 \pm 0.08$ & $\mathrm{SMC}$ & $0.05 \pm 0.02$ & $0.81 \pm 0.11$ & Opt: $67 ; \mathrm{X}: 36$ & $z: 12 ;$ Opt: 13 \\
\hline $060714 \dagger$ & 2.711 & 0.92 & SMC & $0.46 \pm 0.17$ & $0.92 \pm 0.10$ & Opt/X: 5 & $z: 14 ;$ Opt/X: 5 \\
\hline 060904B & 0.703 & $1.11 \pm 0.10$ & SMC & $0.08 \pm 0.08$ & $1.11 \pm 0.12$ & Opt: $86 ; \mathrm{X}: 2$ & $z: 4 ;$ Opt: 2 \\
\hline 060908 & 1.884 & $0.30 \pm 0.03$ & SMC & 0.00 & $0.94 \pm 0.08$ & Opt: 86; X: 2 & $z: 4 ;$ Opt: 2 \\
\hline 070411 & 2.954 & & & & $1.19 \pm 0.14$ & $X: 8$ & $z: 4$ \\
\hline $070802 \dagger$ & 2.454 & 0.61 & MW & $1.20 \pm 0.12$ & $1.11 \pm 0.05$ & Opt/X: 2 & $z: 4 ;$ Opt/X: 15 \\
\hline 070810A & 2.170 & $\ldots$ & & $\cdots$ & $1.14 \pm 0.16$ & $X: 2$ & $z: 16$ \\
\hline 080319B & 0.937 & $0.50 \pm 0.07$ & SMC & 0.15 & $0.98 \pm 0.10$ & Opt/X: 170 & $z: 17 ;$ Opt/X: 18 \\
\hline $080514 \mathrm{~B}+$ & 1.800 & $0.63 \pm 0.02$ & SMC & 0.00 & $1.13 \pm 0.13$ & Opt: 43; X: 33 & $z / \mathrm{Opt} / \mathrm{X}: 19$ \\
\hline $080710 \dagger$ & 0.845 & 1.01 & SMC & $0.00 \pm 0.00$ & $1.01 \pm 0.01$ & Opt/X: 27 & $z: 4 ; \mathrm{Opt} / \mathrm{X}: 20$ \\
\hline 080721 & 2.591 & $\ldots$ & & & $0.99 \pm 0.04$ & $X: 4$ & $z: 4$ \\
\hline $080916 \mathrm{C} \dagger$ & 4.350 & 0.49 & SMC & 0.00 & $0.49 \pm 0.34$ & Opt: 79; X: 67 & $z / \mathrm{Opt} / \mathrm{X}: 21$ \\
\hline 081203A & 2.050 & $\ldots$ & & & $1.06 \pm 0.07$ & $X: 22$ & $z: 22$ \\
\hline 090102 & 1.547 & $0.74 \pm 0.22$ & SMC & $0.12 \pm 0.11$ & $0.77 \pm 0.04$ & Opt: $86 ; \mathrm{X}: 8$ & $z: 23$; Opt: 2 \\
\hline 090323 & 3.568 & $0.65 \pm 0.13$ & SMC & $0.14 \pm 0.04$ & $0.95 \pm 0.13$ & Opt: $97 \mathrm{ks}, \mathrm{X}: 92 \mathrm{ks}$ & $z: 24 ;$ Opt: 25 \\
\hline 090328 & 0.735 & $1.17 \pm 0.17$ & SMC & $0.18 \pm 0.13$ & $1.10 \pm 0.16$ & Opt: $86 ; \mathrm{X}: 130$ & $z: 25 ;$ Opt: 2 \\
\hline 090726 & 2.710 & $\ldots$ & $\ldots$ & & $1.45 \pm 0.15$ & $X: 17$ & $z: 26$ \\
\hline 090902B & 1.822 & $0.73 \pm 0.13$ & SMC & $0.05 \pm 0.05$ & $1.02 \pm 0.11$ & Opt: 86; X: 98 & $z: 24 ;$ Opt: 2 \\
\hline 090926A $\dagger$ & 2.106 & 1.04 & MW & $<0.10$ & $1.04 \pm 0.08$ & Opt/X: 250 & $z: 24 ; \mathrm{Opt} / \mathrm{X}: 27$ \\
\hline
\end{tabular}

Notes. For every GRB we list the redshift, $z$, the properties of the optical SED (spectral slope $\beta_{\text {opt }}$, host extinction in the $V$-band rest frame, $A_{V}^{\text {host }}$, and the corresponding extinction law), the spectral slope in the X-rays, $\beta_{\mathrm{x}}$, and the mean time after the burst, $t_{\text {mid }}$, at which the SED was extracted. The events for which we used joint optical-to-X-ray SEDs are marked with a "†". In these cases the difference in the spectral slope, $\beta_{\mathrm{x}}-\beta_{\text {opt }}$ was either fixed to $1 / 2$ or 0 . Except for GRB $080514 \mathrm{~B}$, these estimates have only one error estimate for $\beta_{\mathrm{opt}}$ and $\beta_{\mathrm{x}}$ together due to the fitting procedure described in the associated papers.

References. (1) Berger \& Becker (2005); (2) Kann et al. (2010); (3) de Pasquale et al. (2007); (4) Fynbo et al. (2009); (5) Schady et al. (2010); (6) Piranomonte et al. (2008); (7) Quimby et al. (2005); (8) Soderberg et al. (2006); (9) Kann et al. (2008); (10) Prochaska et al. (2006); (11) Bloom et al. (2006); (12) Della Valle et al. (2006b); (13) Mangano et al. (2007); (14) Jakobsson et al. (2006); (15) Krühler et al. (2008); (16) Thöne et al. (2007); (17) D'Elia et al. (2009); (18) Racusin et al. (2008); (19) Rossi et al. (2009); (20) Krühler et al. (2009); (21) Greiner et al. (2009a); (22) Kuin et al. (2009); (23) de Ugarte Postigo et al. (2009); (24) Cenko et al. (2010); (25) McBreen et al. (2010); (26) Fatkhullin et al. (2009); (27) Rau et al. (2010) 
A\&A 526, A23 (2011)

Table B.2. Light-curve parameters of the late-time optical and X-ray afterglows of the 27 bursts that entered our sample.

\begin{tabular}{|c|c|c|c|c|c|c|c|c|}
\hline \multirow{2}{*}{ GRB } & \multirow{2}{*}{ Band } & \multicolumn{5}{|c|}{ Light-curve parameters } & \multicolumn{2}{|c|}{ Overlapping time interval } \\
\hline & & $t_{\text {start }}^{\text {late }}(\mathrm{ks})$ & $t_{\text {end }}^{\text {late }}(\mathrm{ks})$ & $\alpha_{1}$ & $\alpha_{2}$ & $t_{\mathrm{b}, \mathrm{jet}}(\mathrm{ks})$ & $t_{\text {start }}(\mathrm{ks})$ & $t_{\text {end }}(\mathrm{ks})$ \\
\hline \multirow{2}{*}{050603} & $\mathrm{O}$ & 33.33 & 196.67 & $\cdots$ & $1.97 \pm 0.06$ & \multirow{2}{*}{$<39.2$} & \multirow{2}{*}{39.2} & \multirow{2}{*}{196.7} \\
\hline & $X$ & 39.21 & 577.04 & & $1.67 \pm 0.05$ & & & \\
\hline \multirow{2}{*}{050801} & $\mathrm{O}$ & 0.25 & 106.27 & $1.19 \pm 0.01$ & $\ldots$ & \multirow{2}{*}{$>106.3$} & \multirow{2}{*}{0.25} & \multirow{2}{*}{106.3} \\
\hline & $X$ & 0.22 & 448.10 & $1.29 \pm 0.08$ & $\ldots$ & & & \\
\hline \multirow{2}{*}{ 050820A } & $\mathrm{O}$ & 23.89 & 612.10 & $1.04 \pm 0.01$ & & & \multirow{2}{*}{23.9} & \multirow{2}{*}{612.1} \\
\hline & $\mathrm{X}$ & 4.64 & 3676.50 & $1.19 \pm 0.02$ & $1.73 \pm 0.16$ & $640.2 \pm 138.7$ & & \\
\hline \multirow{2}{*}{ 050922C } & $\mathrm{O}$ & 4.13 & 606.01 & $1.47 \pm 0.04$ & $\ldots$ & \multirow{2}{*}{$>95.9$} & \multirow{2}{*}{4.1} & \multirow{2}{*}{95.9} \\
\hline & $\mathrm{X}$ & 2.02 & 95.92 & $1.37 \pm 0.03$ & $\ldots$ & & & \\
\hline \multirow{2}{*}{ 051109A } & $\mathrm{O}$ & 17.52 & 265.20 & $1.03 \pm 0.06$ & $\ldots$ & \multirow{2}{*}{$>265.2$} & \multirow{2}{*}{17.5} & \multirow{2}{*}{265.2} \\
\hline & $X$ & 3.46 & 1240.80 & $1.20 \pm 0.01$ & $\ldots$ & & & \\
\hline \multirow{2}{*}{ 051221A } & $\mathrm{O}$ & 4.68 & 445.12 & $0.96 \pm 0.03$ & $\ldots$ & & \multirow{2}{*}{15.2} & \multirow{2}{*}{445.1} \\
\hline & $\mathrm{X}$ & 15.17 & 2271.92 & $1.03 \pm 0.01$ & $1.99 \pm 0.17$ & $318.5 \pm 38.6$ & & \\
\hline \multirow{2}{*}{060418} & $\mathrm{O}$ & 0.12 & 871.05 & $1.192 \pm 0.002$ & $\ldots$ & \multirow{2}{*}{$>537.1$} & & \\
\hline & $\mathrm{X}$ & 4.40 & 537.08 & $1.52 \pm 0.05$ & $\ldots$ & & 4.4 & 537.1 \\
\hline & $\mathrm{O}$ & 7.02 & 715.56 & $0.80 \pm 0.03$ & $\cdots$ & & & \\
\hline 060512 & $X$ & 0.24 & 288.61 & $1.12 \pm 0.05$ & . & $>288.6$ & 7.0 & 288.6 \\
\hline & $\mathrm{O}$ & 51.59 & 1700.19 & $1.05 \pm 0.04$ & $2.45 \pm 0.05$ & $113.1 \pm 2.7$ & & \\
\hline 060614 & $X$ & 41.23 & 1500.57 & $1.07 \pm 0.11$ & $2.31 \pm 0.11$ & $120.0 \pm 12.1$ & 51.6 & 1500.6 \\
\hline & $\mathrm{O}$ & 47.07 & 286.77 & $1.42 \pm 0.18$ & $\ldots$ & $>2868$ & & \\
\hline 060714 & $X$ & 3.07 & 1029.03 & $1.23 \pm 0.03$ & $\ldots$ & $>286.8$ & 47.1 & 286.8 \\
\hline $060904 \mathrm{~B}$ & $\mathrm{O}$ & 3.52 & 163.13 & $1.16 \pm 0.02$ & $\ldots$ & $>1631$ & 36 & 1631 \\
\hline $060904 B$ & $X$ & 3.58 & 391.01 & $1.36 \pm 0.03$ & $\ldots$ & $>103.1$ & 3.0 & 103.1 \\
\hline 060908 & $\mathrm{O}$ & 0.14 & 82.42 & $1.03 \pm 0.01$ & $\ldots$ & $>82.4$ & 0.71 & 82.4 \\
\hline & $\mathrm{X}$ & 0.71 & 483.21 & $1.49 \pm 0.07$ & $\ldots$ & $>82.4$ & & \\
\hline 070411 & $\mathrm{O}$ & 92.09 & 523.12 & $1.29 \pm 0.06$ & $\ldots$ & $>523.1$ & 92.1 & 5231 \\
\hline & $X$ & 0.50 & 664.68 & $1.12 \pm 0.02$ & $\ldots$ & & & (120.1 \\
\hline 070802 & $\mathrm{O}$ & 10.90 & 88.88 & $0.90 \pm 0.16$ & $\ldots$ & $>88.9$ & 10.9 & 88.9 \\
\hline & $X$ & 6.68 & 316.52 & $1.17 \pm 0.09$ & $\ldots$ & & & \\
\hline 070810A & $\mathrm{O}$ & 1.95 & 103.36 & $1.30 \pm 0.10$ & $\ldots$ & $>34.5$ & 1.9 & 34.5 \\
\hline & X & 1.55 & 34.46 & $1.29 \pm 0.07$ & $\cdots$ & & & \\
\hline 080319B & $\begin{array}{l}\mathrm{O} \\
\mathrm{X}\end{array}$ & $\begin{array}{r}0.8 \\
42.66\end{array}$ & $\begin{array}{r}1060 \\
2559.17\end{array}$ & $\begin{array}{c}1.237 \pm 0.002 \\
1.04 \pm 0.05\end{array}$ & $\begin{array}{c}\cdots \\
2.68 \pm 0.36\end{array}$ & $953 \pm 137$ & 42.7 & 1060 \\
\hline
\end{tabular}

Notes. Columns 3 to 7 give the time interval, $t_{\text {start }}^{\text {late }}, t_{\text {stop }}^{\text {late }}$, the pre- and post-jet break decay slopes, $\alpha_{1}, \alpha_{2}$, and the observed jet break time, $t_{\mathrm{b} \text {,jet }}$. The optical and X-ray light curves were independently fitted for every GRB (Sect. 2.2). Owing this, two break times are given for GRB 060614. Within the errors the break was achromatic, making it a good candidate for a jet break. For the other GRBs we state the upper or lower limits on the jet break time with respect to the identification of the dynamical regime shown in Table B.3. The overlapping time interval of the late-time optical and X-ray afterglow is shown in the last two columns. $\dagger$ The optical afterglow light curve of GRB 080721 shows an additional shallow break at $(129 \pm 84) \mathrm{ks}$. The difference in the pre- and post-break decay slope is $\approx 0.25$ in agreement with Kann et al. (2010), which is typical for a cooling break. This break is not a jet break. 
S. Schulze et al.: The circumburst density profile around GRB progenitors: a statistical study

Table B.2. continued.

\begin{tabular}{|c|c|c|c|c|c|c|c|c|}
\hline \multirow{2}{*}{ GRB } & \multirow{2}{*}{ Band } & \multicolumn{5}{|c|}{ Light-curve parameters } & \multicolumn{2}{|c|}{ Overlapping time interval } \\
\hline & & $t_{\text {start }}(\mathrm{ks})$ & $t_{\text {end }}(\mathrm{ks})$ & $\alpha_{1}$ & $\alpha_{2}$ & $t_{\mathrm{b}, \mathrm{jet}}(\mathrm{ks})$ & $t_{\text {start }}(\mathrm{ks})$ & $t_{\text {end }}(\mathrm{ks})$ \\
\hline \multirow{2}{*}{ 080514B } & $\mathrm{O}$ & 37.15 & 174.79 & $1.64 \pm 0.06$ & & \multirow{2}{*}{$>174.8$} & \multirow{2}{*}{37.3} & \multirow{2}{*}{174.8} \\
\hline & $\mathrm{X}$ & 37.30 & 217.27 & $1.54 \pm 0.14$ & $\ldots$ & & & \\
\hline \multirow{2}{*}{080710} & $\mathrm{O}$ & 9.97 & 353.11 & $1.57 \pm 0.02$ & & \multirow{2}{*}{$>349.3$} & \multirow{2}{*}{11.3} & \multirow{2}{*}{349.3} \\
\hline & $\mathrm{X}$ & 11.34 & 349.31 & $1.56 \pm 0.09$ & $\ldots$ & & & \\
\hline \multirow{2}{*}{$080721 \dagger$} & $\mathrm{O}$ & 0.17 & 2641.08 & $1.22 \pm 0.01$ & $1.46 \pm 0.08$ & \multirow{2}{*}{$>1256.8$} & \multirow{2}{*}{29.5} & \multirow{2}{*}{1256.8} \\
\hline & $\mathrm{X}$ & 29.46 & 1256.83 & $1.50 \pm 0.03$ & $\ldots$ & & & \\
\hline \multirow{2}{*}{ 080916C } & $\mathrm{O}$ & 97.49 & 377.94 & $1.40 \pm 0.10$ & $\ldots$ & \multirow{2}{*}{$>377.9$} & \multirow{2}{*}{97.5} & \multirow{2}{*}{377.9} \\
\hline & $\mathrm{X}$ & 65.03 & 1306.33 & $1.29 \pm 0.08$ & & & & \\
\hline \multirow{2}{*}{ 081203A } & $\mathrm{O}$ & 6.60 & 301.54 & & $1.72 \pm 0.01$ & & \multirow{2}{*}{8.9} & \multirow{2}{*}{301.5} \\
\hline & $X$ & 8.91 & 345.00 & $1.13 \pm 0.01$ & $1.93 \pm 0.06$ & $8.9 \pm 0.9$ & & \\
\hline \multirow{2}{*}{090102} & $\mathrm{O}$ & 15.76 & 263.68 & $1.50 \pm 0.03$ & $\ldots$ & \multirow{2}{*}{$>263.6$} & \multirow{2}{*}{15.8} & \multirow{2}{*}{263.7} \\
\hline & $\mathrm{X}$ & 0.95 & 688.48 & $1.43 \pm 0.02$ & & & & \\
\hline \multirow{2}{*}{090323} & $\mathrm{O}$ & 96.81 & 1142.30 & $\ldots$ & $1.88 \pm 0.01$ & \multirow{2}{*}{$<96.8$} & \multirow{2}{*}{96.8} & \multirow{2}{*}{1084.8} \\
\hline & $\mathrm{X}$ & 70.47 & 1084.77 & $\ldots$ & $1.56 \pm 0.10$ & & & \\
\hline 090328 & $\mathrm{O}$ & 57.28 & 1007.14 & .. & $1.78 \pm 0.04$ & \multirow{2}{*}{$<57.3$} & 57.3 & 924.6 \\
\hline & $\begin{array}{l}\lambda \\
\mathrm{O}\end{array}$ & $\begin{array}{l}0.15 \\
1.40\end{array}$ & $\begin{array}{r}924.60 \\
10.00\end{array}$ & $\begin{array}{c}\cdots \\
097+010\end{array}$ & $1.68 \pm 0.09$ & & & \\
\hline 090726 & $\mathrm{X}$ & 3.61 & 66.43 & $1.34 \pm 0.04$ & $\cdots$ & 0.0 & 3.6 & 10.0 \\
\hline $090902 \mathrm{~B}$ & $\mathrm{O}$ & 45.64 & 1171.46 & $0.97 \pm 0.02$ & $\ldots$ & $>1171.5$ & 456 & 11715 \\
\hline $030702 \mathrm{D}$ & X & 45.21 & 1456.69 & $1.33 \pm 0.03$ & $\ldots$ & $>11 / 1.5$ & 45.6 & $11 / 1.5$ \\
\hline 090926A & $\mathrm{O}$ & 254.24 & 2070.84 & $1.74 \pm 0.02$ & $\ldots$ & $>1803.5$ & 254.2 & 1803.5 \\
\hline OYOYZOA & $X$ & 51.49 & 1803.53 & $1.53 \pm 0.07$ & $\ldots$ & 31005.0 & 234.2 & 1005.0 \\
\hline
\end{tabular}


Table B.3. Identification of the light-curve segments and the circumburst medium.

\begin{tabular}{|c|c|c|c|c|c|c|c|}
\hline \multirow{2}{*}{ GRB } & \multirow{2}{*}{ Closure relation } & \multirow{2}{*}{$\alpha_{\mathrm{x}}-\alpha_{\mathrm{opt}}$} & \multirow{2}{*}{$\beta_{\mathrm{x}}-\beta_{\mathrm{opt}}$} & \multirow{2}{*}{\multicolumn{2}{|c|}{ Conclusion }} & \multicolumn{2}{|c|}{ Literature } \\
\hline & & & & & & ID & Reference \\
\hline 050603 & $\begin{array}{l}O:- \\
X: j 1 / 2 a, j 2 b\end{array}$ & $-0.30 \pm 0.08$ & - & $\begin{array}{l}O: j 1 b \\
X: j 2 b\end{array}$ & Wind & & \\
\hline 050801 & $\begin{array}{l}\text { O: } \\
\text { X: S1a }\end{array}$ & $0.10 \pm 0.08$ & $0.20 \pm 0.35$ & $\begin{array}{l}\text { O: S1a } \\
\text { X: S1a }\end{array}$ & ISM & $\begin{array}{l}\text { O: S1a } \\
\text { X: S1a }\end{array}$ & 1 \\
\hline 050820A & $\begin{array}{l}\text { O: S1a } \\
X_{1}: S 2 \\
X_{2}: J 2, j 2 a / b\end{array}$ & $0.15 \pm 0.02$ & $0.37 \pm 0.04$ & $\begin{array}{l}\mathrm{O}: \mathrm{S} 1 \mathrm{a} \\
\mathrm{X}_{1}: \mathrm{S} 2 \\
\mathrm{X}_{2}: \mathrm{j} 2 \mathrm{a}\end{array}$ & ISM & $\begin{array}{l}\text { O: S1a } \\
\mathrm{X}_{1}: \mathrm{S} 2 \\
\mathrm{X}_{2}:-\end{array}$ & 2 \\
\hline 050922C & $\begin{array}{l}\text { O: S1a } \\
\text { X: S1a }\end{array}$ & $-0.10 \pm 0.05$ & $0.00 \pm 0.05$ & $\begin{array}{l}\text { O: S1a } \\
\text { X: S1a }\end{array}$ & ISM & & \\
\hline 051109A & $\begin{array}{l}\text { O: } \\
\text { X: S1a, S2 }\end{array}$ & $0.17 \pm 0.06$ & $0.50 \pm 0.04$ & $\begin{array}{l}\text { O: S1a } \\
\text { X: S2 }\end{array}$ & ISM & & \\
\hline $051221 \mathrm{~A}$ & $\begin{array}{l}\text { O:- } \\
X_{1}: S 1 a, S 2 \\
X_{2}: J 2, j 1 / 2 a, j 1 / 2 b\end{array}$ & $0.07 \pm 0.03$ & - & $\begin{array}{l}\mathrm{O}: \mathrm{S} 2 \\
\mathrm{X}_{1}: \mathrm{S} 2 \\
\mathrm{X}_{2}: \mathrm{J} 2\end{array}$ & - & $\mathrm{X}_{1}: \mathrm{S} 2$ & 3 \\
\hline 060418 & $\begin{array}{l}\text { O: S1a } \\
\text { X: S1a/b, S2 }\end{array}$ & $0.33 \pm 0.05$ & $0.29 \pm 0.19$ & $\begin{array}{l}\text { O: S1a } \\
\text { X: S2 }\end{array}$ & ISM & & \\
\hline 060512 & $\begin{array}{l}\text { O:- } \\
\text { X: S1a, S2 }\end{array}$ & $0.32 \pm 0.06$ & - & $\begin{array}{l}\text { O: S1a } \\
\text { X: S2 }\end{array}$ & ISM & & \\
\hline 060614 & $\begin{array}{l}\mathrm{O}_{1}: \mathrm{S} 1 \mathrm{a}, \mathrm{S} 2 \\
\mathrm{X}_{1}: \mathrm{S} 1 \mathrm{a}, \mathrm{S} 2 \\
\mathrm{O}_{2}: \mathrm{J} 1, \mathrm{j} 1 \mathrm{~b} \\
\mathrm{X}_{2}: \mathrm{J} 1 / 2, \mathrm{j} 1 \mathrm{a} / \mathrm{b}\end{array}$ & $\begin{array}{r}0.02 \pm 0.12 \\
-0.14 \pm 0.12\end{array}$ & $\begin{array}{l}0.00 \pm 0.11 \\
0.00 \pm 0.11\end{array}$ & $\begin{array}{l}\mathrm{O}_{1}: \mathrm{S} 1 \mathrm{a} \\
\mathrm{X}_{1}: \mathrm{S} 1 \mathrm{a} \\
\mathrm{O}_{2}: \mathrm{J} 1 \\
\mathrm{X}_{2}: \mathrm{J} 1\end{array}$ & ISM & $\begin{array}{l}\mathrm{X}_{1}: \mathrm{S} 1 \mathrm{a} \\
\mathrm{X}_{2}: \mathrm{J} 1\end{array}$ & 4 \\
\hline 060714 & $\begin{array}{l}\text { O: S1a/b, S2 } \\
\text { X: S1a, S2 }\end{array}$ & $-0.19 \pm 0.18$ & $0.00 \pm 0.10$ & $\begin{array}{l}\text { O: S1a } \\
\text { X: S1a }\end{array}$ & ISM & & \\
\hline 060904B & $\begin{array}{l}\text { O: S2 } \\
\text { X: S1a, S2 }\end{array}$ & $0.20 \pm 0.04$ & $0.00 \pm 0.16$ & $\begin{array}{l}\mathrm{O}: \mathrm{S} 2 \\
\mathrm{X}: \mathrm{S} 2\end{array}$ & - & & \\
\hline 060908 & $\begin{array}{l}\text { O:- } \\
\text { X: S1a }\end{array}$ & $0.46 \pm 0.07$ & $0.64 \pm 0.09$ & $\begin{array}{l}\text { O: S1a } \\
\text { X: S2 }\end{array}$ & ISM & & \\
\hline 070411 & $\begin{array}{l}\mathrm{O}:- \\
\mathrm{X}: \mathrm{S} 2\end{array}$ & $-0.17 \pm 0.06$ & - & $\begin{array}{l}\text { O: S1b } \\
\text { X: S2 }\end{array}$ & Wind & & \\
\hline
\end{tabular}

Notes. Following the scheme in Sect. 3.1.2, different criteria were applied to find a consistent description of the late-time optical and X-ray afterglow data to reveal the nature of the circumburst medium. The light-curve segments were labelled following Panaitescu (2007, for the designated ID see also Table 2) $\left((\mathrm{S}, \mathrm{J}, \mathrm{j})=(\right.$ spherical expansion, jet with sideways expansion, jet without lateral spreading $),(1,2)=\left(v<v_{\mathrm{c}}, v>v_{\mathrm{c}}\right),(\mathrm{a}$, b)=(ISM, wind); see also Table 2). If there was a break in the light curve, the segments were tagged with the subscript 1 and 2 . The second column lists the light-curve segments and all closure relations that agree with the observational data within $3 \sigma$. The third and fourth column show the corresponding measured differences in the temporal and spectral slopes, if available. Their uncertainties are $1 \sigma$ errors. If a criterion could not be applied or a solution was not found, we crossed the field out. The fifth and sixth columns show the conclusion based on all criteria, i.e., the segment label and the type of circumburst medium. The second last column summarises the results from the literature.

References. (1) Rykoff et al. (2006); (2) Cenko et al. (2006); (3) Burrows et al. (2006); (4) Mangano et al. (2007) 
Table B.3. continued.

\begin{tabular}{|c|c|c|c|c|c|c|c|}
\hline \multirow{3}{*}{$\frac{\text { GRB }}{070802}$} & \multirow{3}{*}{$\begin{array}{l}\text { Closure relation } \\
\mathrm{O}: \mathrm{S} 1 \mathrm{a} / \mathrm{b}, \mathrm{S} 2 \\
\mathrm{X}: \mathrm{S} 2\end{array}$} & \multirow{3}{*}{$\frac{\alpha_{\mathrm{x}}-\alpha_{\mathrm{opt}}}{0.27 \pm 0.18}$} & \multirow{3}{*}{$\frac{\beta_{\mathrm{x}}-\beta_{\mathrm{opt}}}{0.50 \pm 0.05}$} & \multirow{2}{*}{\multicolumn{2}{|c|}{ Conclusion }} & \multicolumn{2}{|l|}{ Literature } \\
\hline & & & & & & ID & Reference \\
\hline & & & & $\begin{array}{l}\text { O: S1a } \\
\text { X: S2 }\end{array}$ & ISM & $\begin{array}{l}\text { O: S2 } \\
\text { X: S2 }\end{array}$ & 5 \\
\hline 070810A & $\begin{array}{l}\text { O: - } \\
\text { X: S1a, S2 }\end{array}$ & $-0.01 \pm 0.12$ & - & $\begin{array}{l}\text { O: S1a } \\
\mathrm{X}: \mathrm{S} 2\end{array}$ & ISM & & \\
\hline 080319B & $\begin{array}{l}\text { O: S1b } \\
X_{1}: S 1 a, S 2 \\
X_{2}:\end{array}$ & $-0.20 \pm 0.05$ & $0.48 \pm 0.12$ & $\begin{array}{l}\text { O: S1b } \\
\mathrm{X}_{1}: \mathrm{S} 2 \\
\mathrm{X}_{2}:-\end{array}$ & Wind & $\begin{array}{l}\mathrm{O}: \mathrm{S} 1 \mathrm{~b} \\
\mathrm{X}_{1}: \mathrm{S} 2 \\
\mathrm{X}_{2}: \mathrm{J} 2\end{array}$ & 6 \\
\hline 080514B & $\begin{array}{l}\mathrm{O}: \mathrm{S} 1 \mathrm{~b} \\
\mathrm{X}: \mathrm{S} 1 \mathrm{a} / \mathrm{b}, \mathrm{S} 2\end{array}$ & $-0.10 \pm 0.14$ & $0.50 \pm 0.13$ & $\begin{array}{l}\text { O: S1b } \\
X: S 2\end{array}$ & Wind & $\begin{array}{l}\text { O: S1b } \\
\text { X: S2 }\end{array}$ & 7 \\
\hline 080710 & $\begin{array}{l}\mathrm{O}: \mathrm{S} 1 \mathrm{a}, \mathrm{j} 2 \mathrm{~b} \\
\mathrm{X}: \mathrm{S} 1 \mathrm{a}, \mathrm{j} 2 \mathrm{a} / \mathrm{b} \\
\mathrm{O}_{1}:-\end{array}$ & $-0.01 \pm 0.09$ & $0.00 \pm 0.01$ & $\begin{array}{l}\text { O: S1a } \\
\text { X: S1a } \\
\mathrm{O}_{1}: \text { S1a }\end{array}$ & ISM & $\begin{array}{l}\text { O: S1a } \\
X: S 1 a \\
\left.\text { 1) } \mathrm{O}_{1}: \mathrm{S} 1 \mathrm{a} ; 2\right) \mathrm{O}: \mathrm{S} 1 \mathrm{a}\end{array}$ & 8 \\
\hline 080721 & $\begin{array}{l}\mathrm{O}_{2}:- \\
\mathrm{X}: \mathrm{S} 2\end{array}$ & $0.04 \pm 0.09$ & - & $\begin{array}{l}\mathrm{O}_{2}: \mathrm{S} 2 \\
\mathrm{X}: \mathrm{S} 2\end{array}$ & ISM & $\begin{array}{l}\text { 1) } \mathrm{O}_{2}: \mathrm{S} 2 \\
\text { 1) } \mathrm{X}:- \text {; 2) X: S1a }\end{array}$ & 1) 9 ;2) 10 \\
\hline 080916C & $\begin{array}{l}\mathrm{O}: \\
\mathrm{X}:\end{array}$ & $-0.11 \pm 0.13$ & $0.00 \pm 0.34$ & $\begin{array}{l}\mathrm{O}: \mathrm{S} 1 \mathrm{~b} \\
\mathrm{X}: \mathrm{S} 1 \mathrm{~b}\end{array}$ & Wind & $\begin{array}{l}\mathrm{O}: \mathrm{S} 1 \mathrm{a} / \mathrm{b} \\
\mathrm{X}: \mathrm{S} 1 \mathrm{a} / \mathrm{b}\end{array}$ & 11 \\
\hline 081203A & $\begin{array}{l}\mathrm{X}_{1}: \mathrm{S} 2 \\
\mathrm{O}:- \\
\mathrm{X}_{2}: \mathrm{J} 2, \mathrm{j} 2 \mathrm{a} / \mathrm{b}\end{array}$ & $0.21 \pm 0.06$ & - & $\begin{array}{l}\mathrm{X}_{1}: \mathrm{S} 2 \\
\mathrm{O}: \mathrm{j} 1 \mathrm{a} \\
\mathrm{X}_{2}: \mathrm{j} 2 \mathrm{a}\end{array}$ & ISM & & \\
\hline 090102 & $\begin{array}{l}\mathrm{O}: \\
\mathrm{X}:\end{array}$ & $0.07 \pm 0.04$ & $0.02 \pm 0.22$ & $\begin{array}{l}\text { O: S1a } \\
\text { X: S1a }\end{array}$ & ISM & $\begin{array}{l}\text { O: S1a } \\
\text { X: S1a }\end{array}$ & 12 \\
\hline 090323 & $\begin{array}{l}\mathrm{O}: \mathrm{S} 1 \mathrm{~b}, \mathrm{j} 1 \mathrm{a} / \mathrm{b} \\
\mathrm{X}: \mathrm{S} 1 \mathrm{a} / \mathrm{b}, \mathrm{S} 2, \mathrm{j} 1 / 2 \mathrm{a}, \mathrm{j} 2 \mathrm{~b}\end{array}$ & $-0.32 \pm 0.10$ & $0.30 \pm 0.18$ & $\begin{array}{l}O: j 1 b \\
X: j 2 b\end{array}$ & Wind & $\begin{array}{l}\text { 1) } \mathrm{O}: \mathrm{J} 1, \mathrm{j} 1 \mathrm{a} / \mathrm{b} \text {; 2) O: S1b } \\
\text { 1) } \mathrm{X}:- \text {-; 2) } \mathrm{X}: \mathrm{S} 1 \mathrm{~b}\end{array}$ & 1) 13 ; 2) 14 \\
\hline 090328 & $\begin{array}{l}\mathrm{O}: \mathrm{S} 1 \mathrm{a} / \mathrm{b}, \mathrm{S} 2, \mathrm{j} 1 \mathrm{a}, \mathrm{j} 2 \mathrm{a} / \mathrm{b} \\
\mathrm{X}: \mathrm{S} 1 \mathrm{a} / \mathrm{b}, \mathrm{S} 2, \mathrm{j} 1 \mathrm{a}, \mathrm{j} 2 \mathrm{a} / \mathrm{b}\end{array}$ & $-0.10 \pm 0.09$ & $-0.07 \pm 0.21$ & $\begin{array}{l}O: j 2 a \\
X: j 2 a\end{array}$ & ISM & $\begin{array}{l}\text { O: J1 } \\
\text { X: J1 }\end{array}$ & 13 \\
\hline 090726 & $\begin{array}{l}\mathrm{O}:- \\
\mathrm{X}: \mathrm{S} 2\end{array}$ & $0.37 \pm 0.10$ & - & $\begin{array}{l}\text { O: S1a } \\
\text { X: S2 }\end{array}$ & ISM & $\begin{array}{l}\text { O: S1a } \\
\text { X: S2 }\end{array}$ & 15 \\
\hline 090902B & $\begin{array}{l}\text { O: S1a, S2 } \\
\text { X: S1a, S2 }\end{array}$ & $0.36 \pm 0.04$ & $0.29 \pm 0.17$ & $\begin{array}{l}\text { O: S1a } \\
\text { X: S2 }\end{array}$ & ISM & $\begin{array}{l}\text { O: S1a } \\
\text { X: S2 }\end{array}$ & 13,14 \\
\hline 090926A & $\begin{array}{l}\text { O: S1a/b } \\
\text { X: S1a }\end{array}$ & $-0.21 \pm 0.07$ & $0.00 \pm 0.08$ & $\begin{array}{l}\text { O: S1a } \\
\text { X: S1a }\end{array}$ & ISM & $\begin{array}{l}\text { 1) O: } \mathrm{S} 2 \text {; 2) O: } \mathrm{S} 1 \mathrm{a} \\
\text { 1) } \mathrm{X}: \mathrm{S} 2 \text {; 2) X: S1a }\end{array}$ & 1) $14:$ 2) 16 \\
\hline
\end{tabular}

References. (5) Krühler et al. (2008); (6) Racusin et al. (2008); (7) Rossi et al. (2009); (8) Krühler et al. (2009); (9) Kann et al. (2010); (10) Starling et al. (2009); (11) Greiner et al. (2009b); (12) Gendre et al. (2010); (13) McBreen et al. (2010); (14) Cenko et al. (2010); (15) Šimon et al. (2010); (16) Rau et al. (2010) 


\section{Appendix C: Figures of the light curve fits}
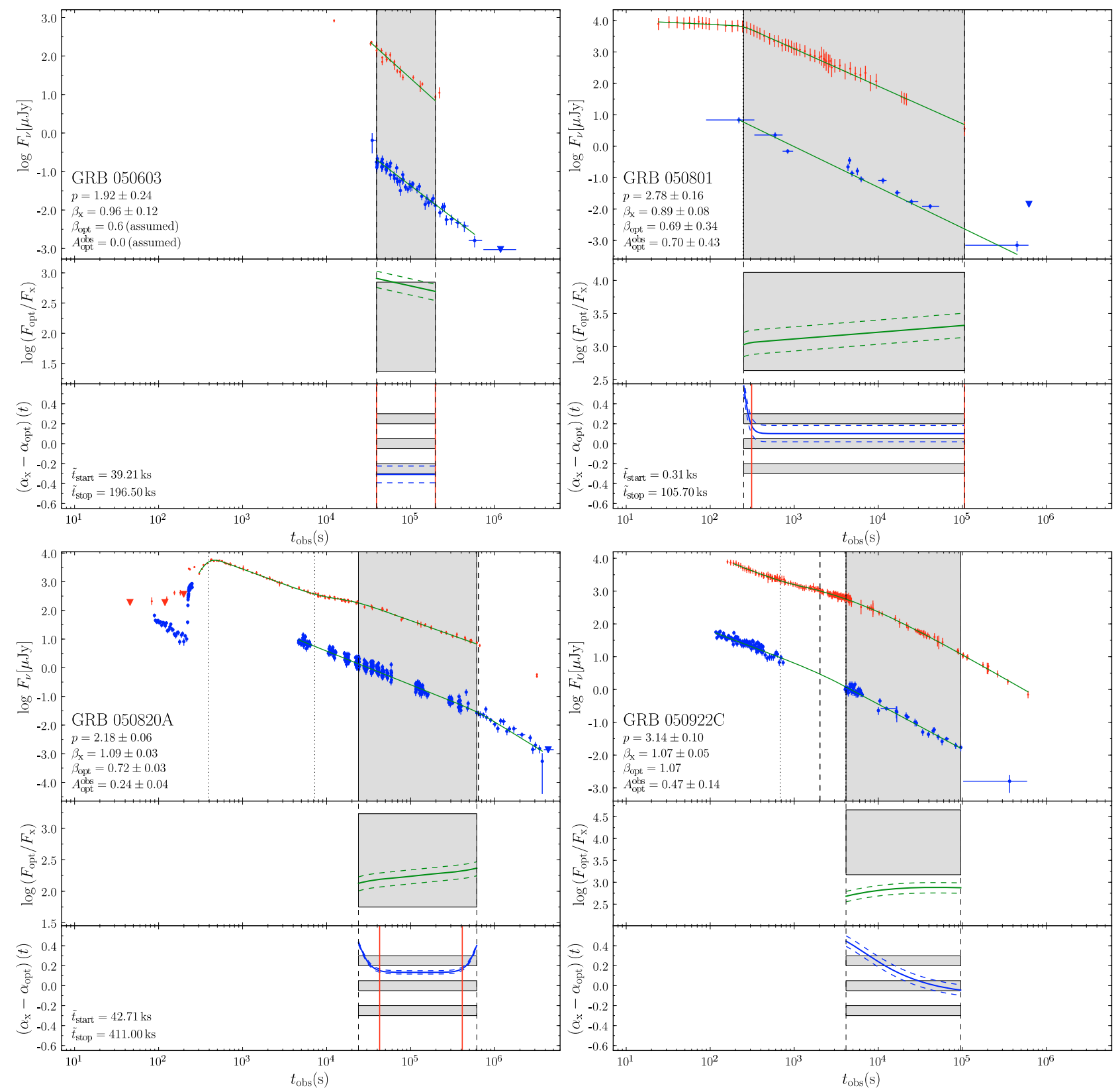

Fig. C.1. Optical and X-ray afterglow light curves of the 27 bursts that entered our sample. Upper panel: the optical data in the $R_{\mathrm{c}}$ band are shown as dots and the X-ray data at $1.73 \mathrm{keV}$ as bigger dots with an error bar in time. The light-curve fits are over-plotted. Upper limits are shown as downwards-pointing triangles. The grey box is the overlapping time interval of the late-time evolution. Vertical dotted and dashed lines indicate breaks in the optical and X-ray band. Information on the SEDs are shown in the bottom left (see also Table B.1). The given extinction, $A_{\mathrm{opt}}^{\text {host }}$, is the observed host-extinction in the $R_{\mathrm{c}}$ band based on the deduced host extinction in the $V$-band, $A_{V}^{\text {host }}$. Additionally, we deduced the electron index, $p$, from $\beta_{\mathrm{x}}$. The electron index is either $p=2 \beta$ if $v_{\mathrm{c}}<v_{\mathrm{x}}$ or $p=2 \beta+1$ if $v_{\mathrm{c}}>v_{\mathrm{x}}$ (e.g., Zhang \& Mészáros 2004). Its error was computed by propagating the uncertainty in $\beta_{\mathrm{x}}$. Middle panel: the flux density ratio between the optical and X-ray afterglow is shown as a solid line and its error as a dashed line for the shared time interval of the late-time evolution. The grey box represents the allowed parameter space of the flux density ratio (Table 1). The upper boundary is the expected flux density ratio for $v_{\mathrm{c}} \leq v_{\mathrm{opt}}$, while the lower one shows the expected ratio for $v_{\mathrm{c}} \geq v_{\mathrm{x}}$. If the cooling break is in between the optical and the X-ray bands, the expected flux-density ratio lies be in between these boundaries. The expected flux density ratio depends on the electron index. Not all bursts could be corrected for host extinction. The error on the electron index was neither propagated into the error of the expected nor of the observed flux-density ratio. Lower panel: the first logarithmic derivative of the flux-density ratio, $\left(\alpha_{\mathrm{x}}-\alpha_{\mathrm{opt}}\right)(t)$, is shown as a solid curve and its error is plotted as a dashed line. For $t / t_{\text {break }} \neq 1$, the first logarithmic derivative is identical to the difference in the decay slopes obtained from the light-curve fit (asymptotic values). Usually breaks in the light curves tend to be smooth instead of sharp. Because of this, the first logarithmic derivative deviates from the asymptotic value close to a break depending on the smoothness of the break. Two solid lines are plotted to highlight the time interval when the asymptotic decay slopes were reached within $1 \sigma$. The precise values are shown on the left and in Table 3 . Within $3 \sigma$, the asymptotic difference in the decay slopes agrees either with $+1 / 4,0,-1 / 4$ depending on the spectral and dynamical regime and the circumburst density profile. Furthermore, an envelope is drawn around expected values, $+1 / 4,0$, $-1 / 4$, with a width of 0.1 to guide the eye. 
S. Schulze et al.: The circumburst density profile around GRB progenitors: a statistical study
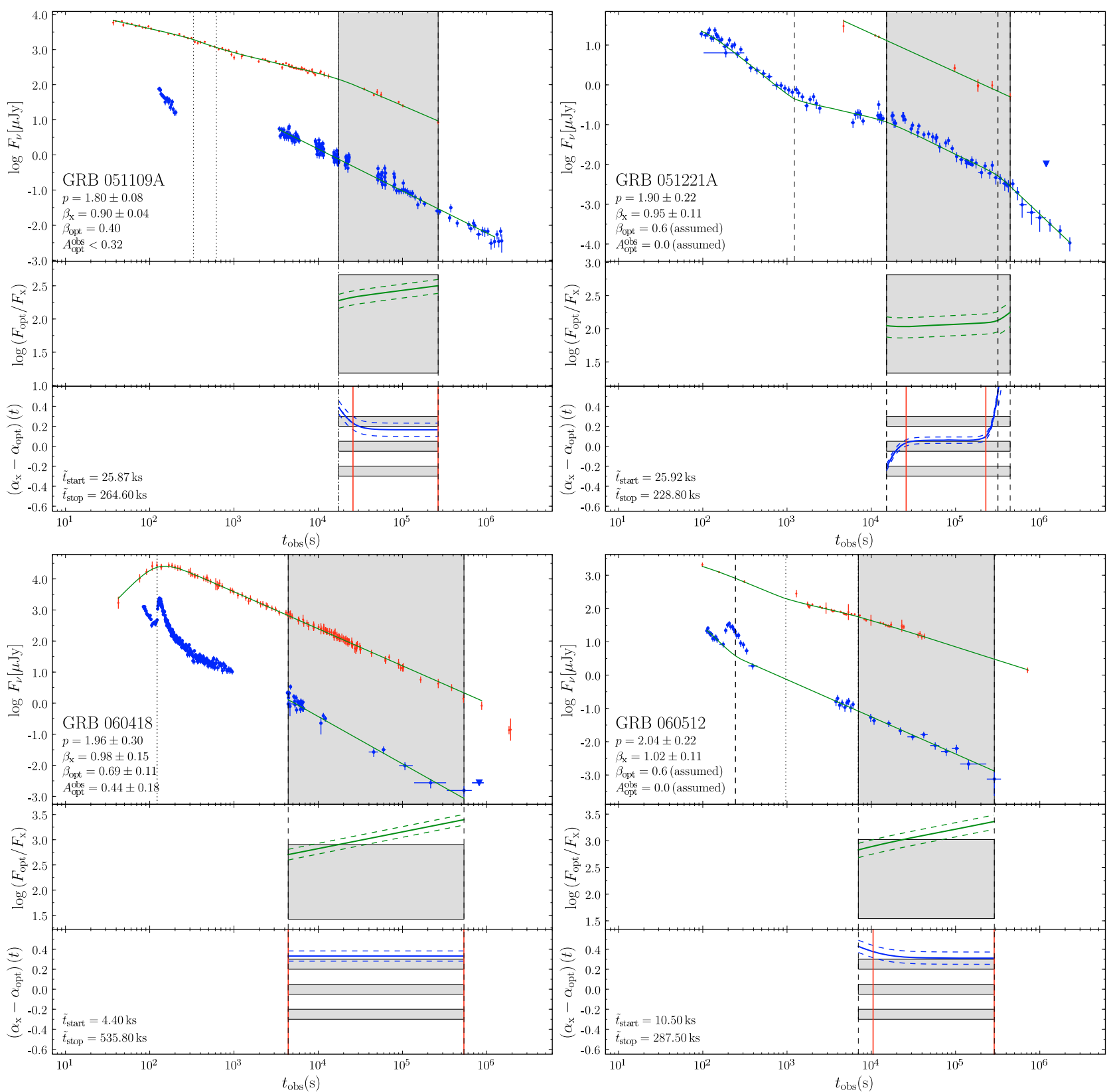

Fig. C.1. continued. 
A\&A 526, A23 (2011)
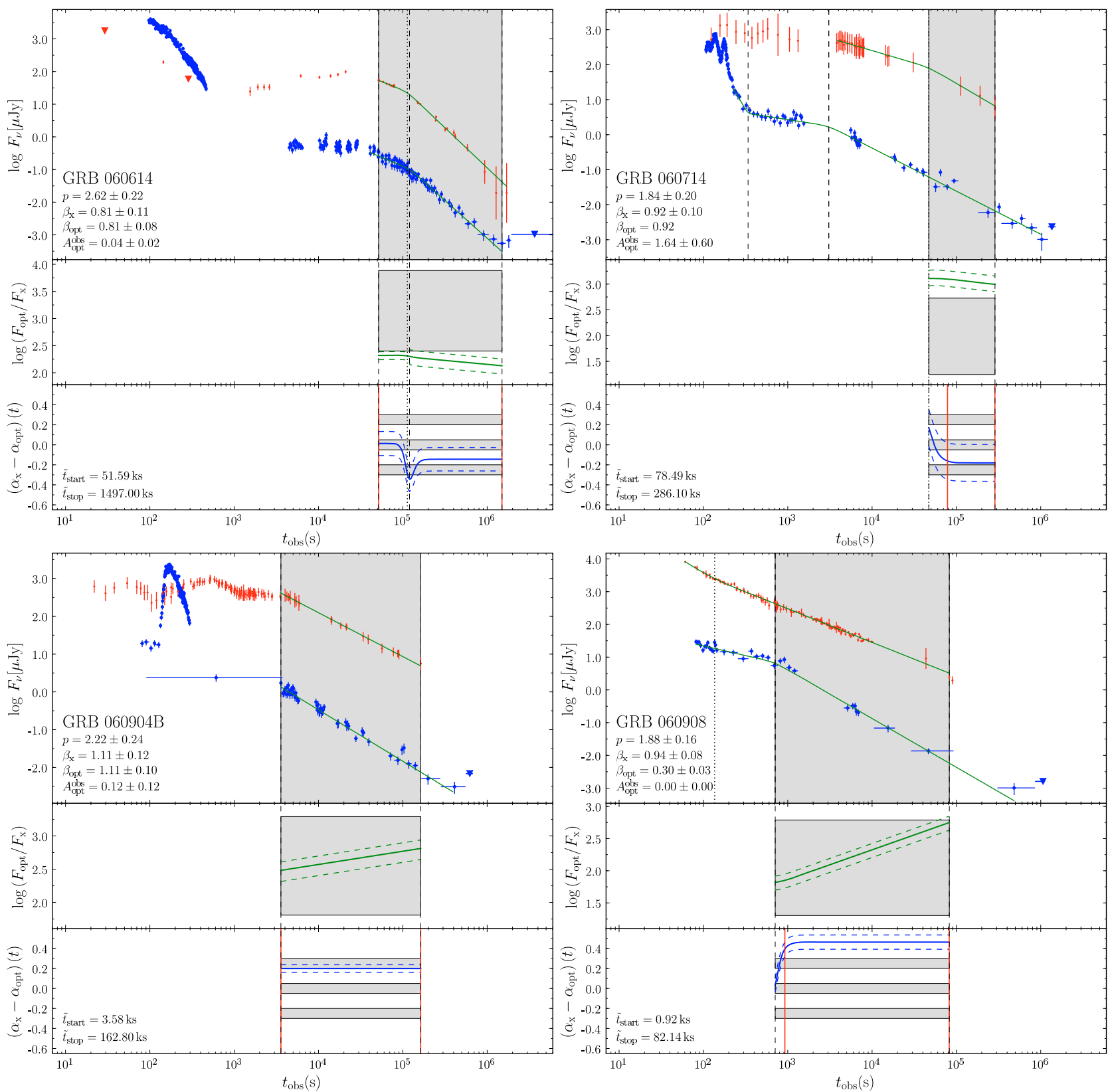

Fig. C.1. continued. 
S. Schulze et al.: The circumburst density profile around GRB progenitors: a statistical study
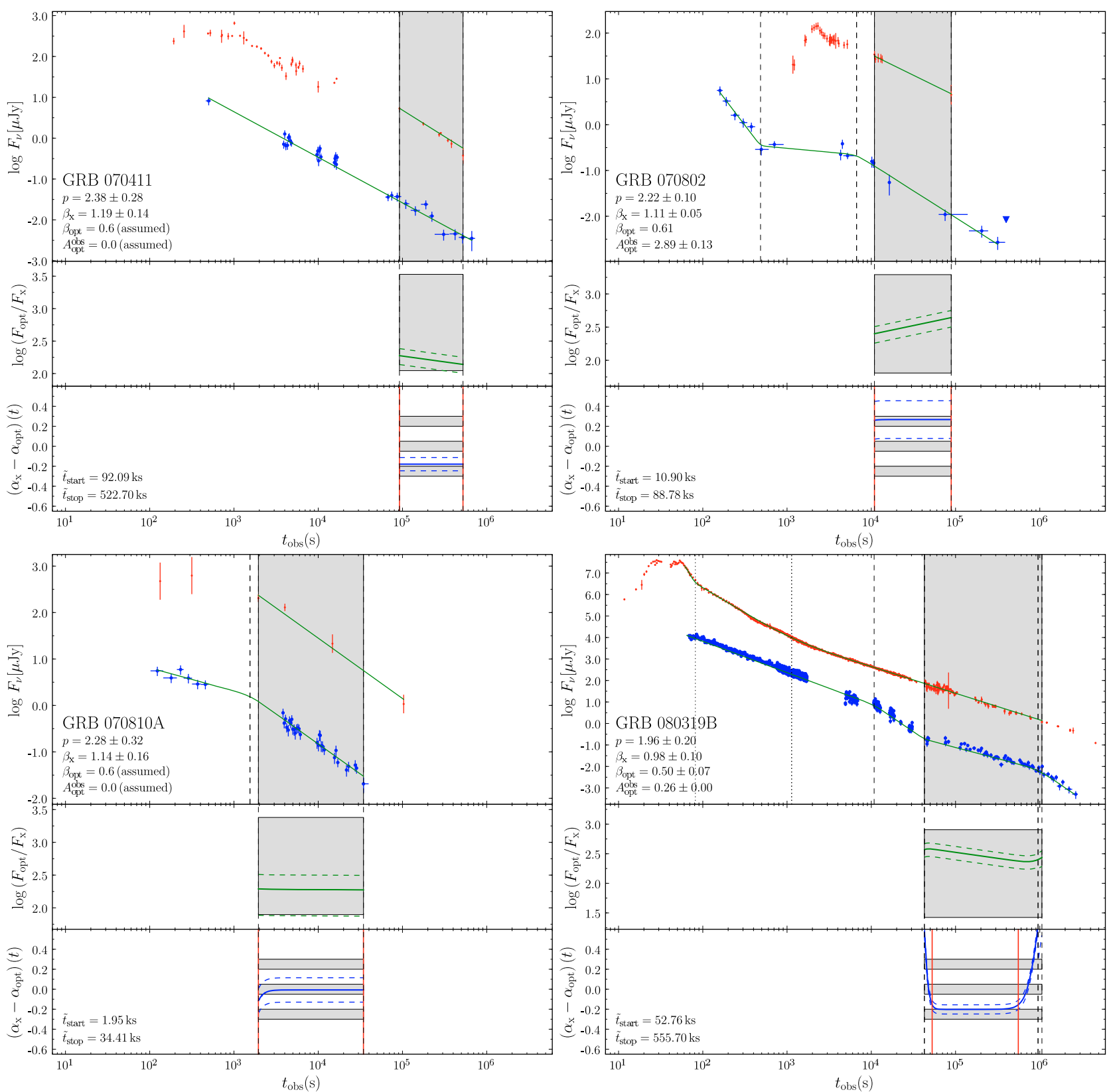

Fig. C.1. continued. 
A\&A 526, A23 (2011)
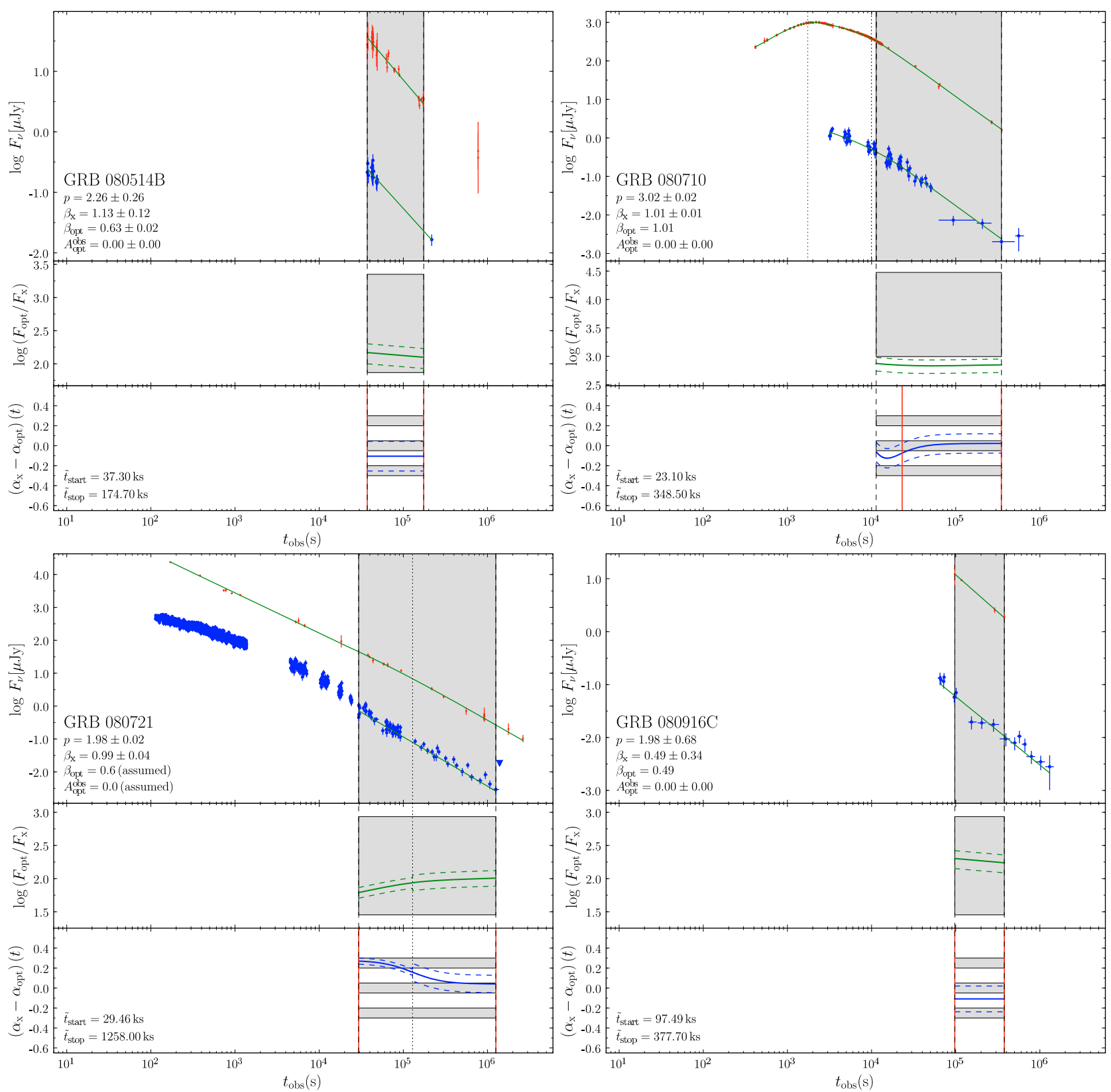

Fig. C.1. continued. 
S. Schulze et al.: The circumburst density profile around GRB progenitors: a statistical study
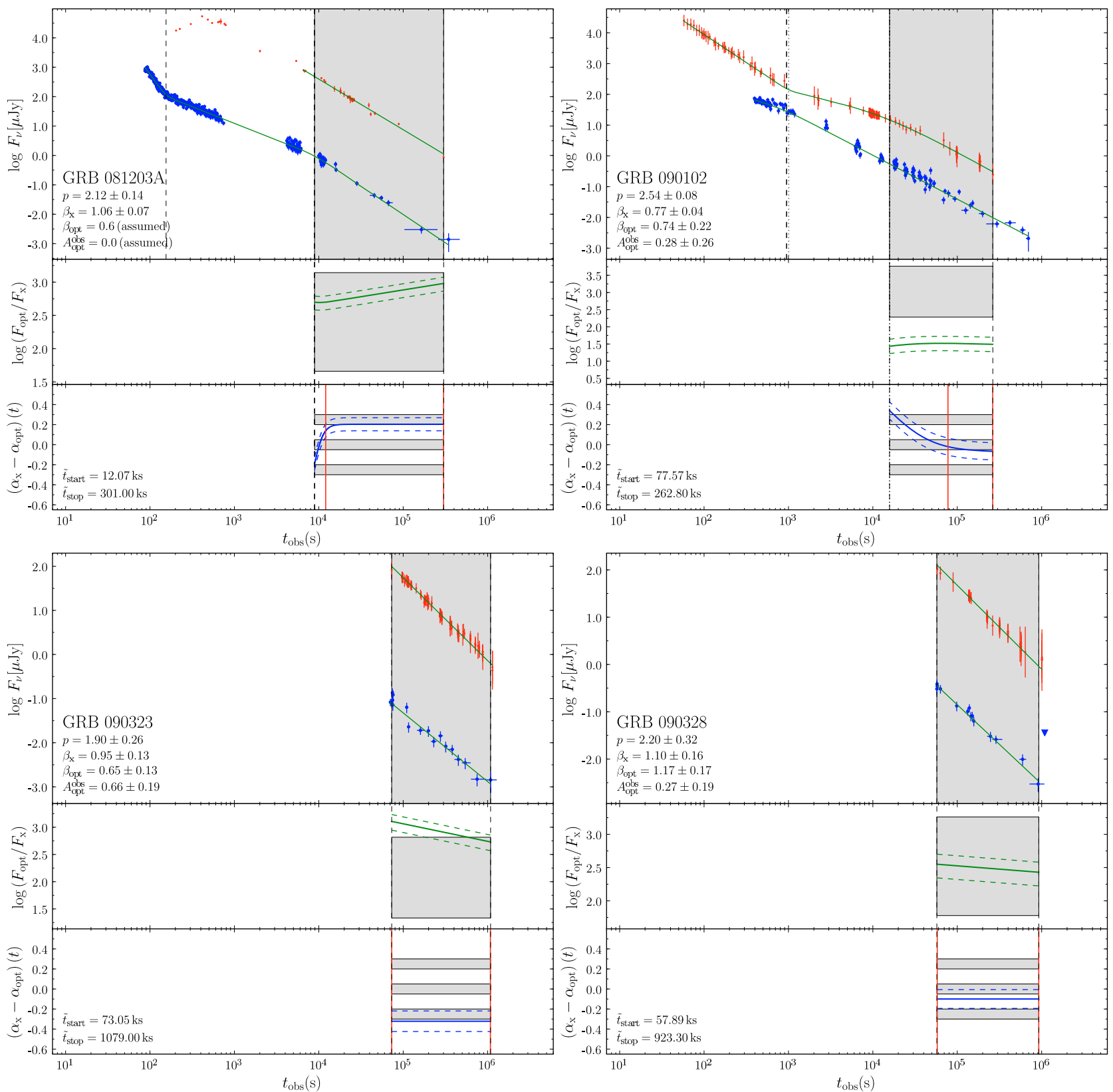

Fig. C.1. continued. 
A\&A 526, A23 (2011)
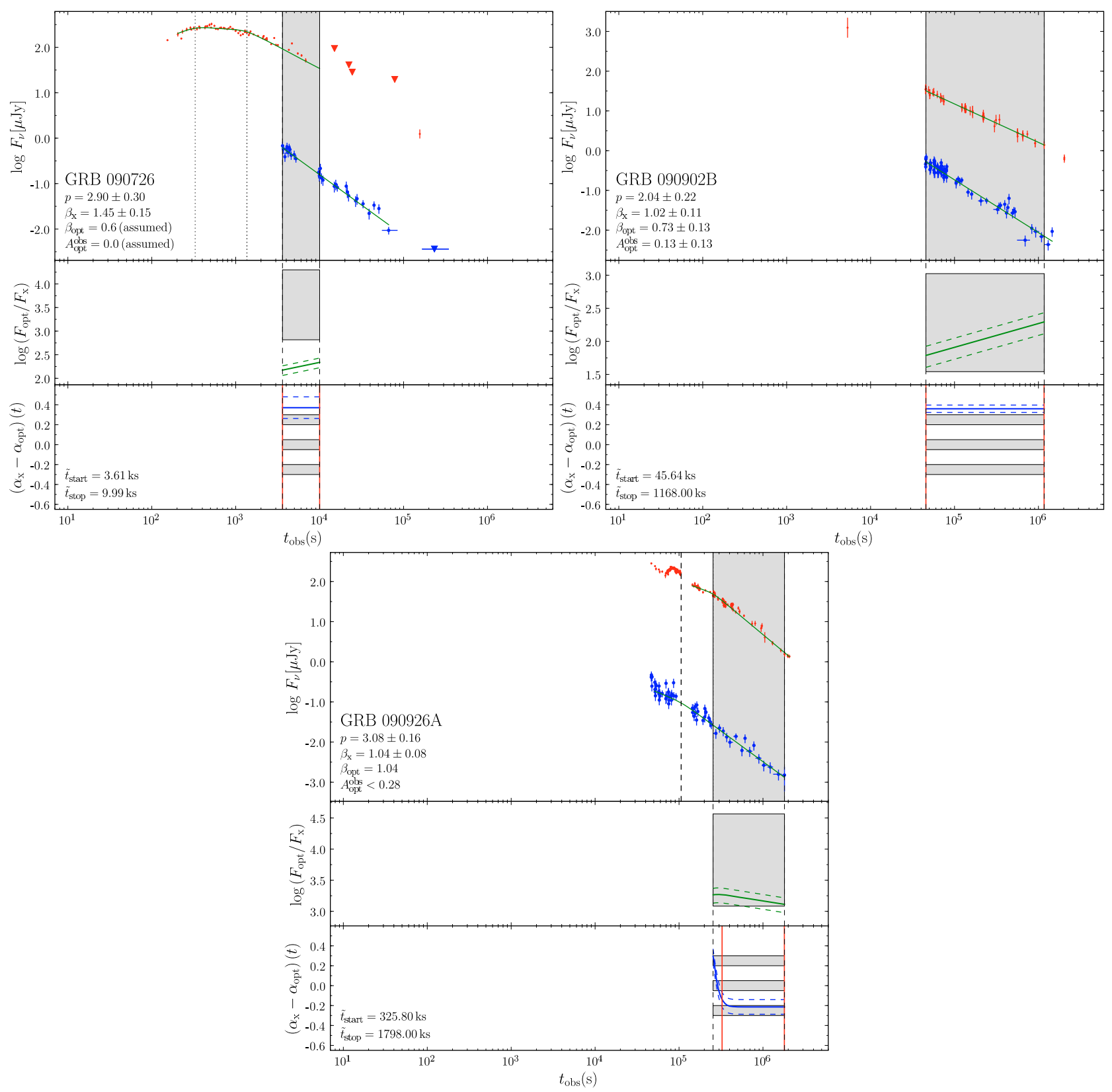

Fig. C.1. continued. 